\title{
SPECTRAL APPROXIMATION OF THE HELMHOLTZ EQUATION WITH HIGH WAVE NUMBERS*
}

\author{
JIE SHEN $^{\dagger}$ AND LI-LIAN WANG ${ }^{\dagger}$
}

\begin{abstract}
A complete error analysis is performed for the spectral-Galerkin approximation of a model Helmholtz equation with high wave numbers. The analysis presented in this paper does not rely on the explicit knowledge of continuous/discrete Green's functions and does not require any mesh condition to be satisfied. Furthermore, new error estimates are also established for multidimensional radial and spherical symmetric domains. Illustrative numerical results in agreement with the theoretical analysis are presented.
\end{abstract}

Key words. Helmholtz equation, high wave numbers, spectral-Galerkin approximation, error analysis

AMS subject classifications. $65 \mathrm{~N} 35,65 \mathrm{~N} 22,35 \mathrm{~J} 05,65 \mathrm{~F} 05$

DOI. $10.1137 / 040607332$

1. Introduction. Time harmonic wave propagations appear in many applications, e.g., wave scattering and transmission, noise reduction, fluid-solid interaction, and sea and earthquake wave propagation. In many situations, time harmonic wave propagations are governed by the following Helmholtz equation in an exterior domain with the so-called Sommerfeld radiation boundary condition:

$$
\begin{aligned}
& -\Delta u-k^{2} u=f \quad \text { in } R^{n} \backslash D, \\
& \left.u\right|_{\partial D}=0, \quad \partial_{r} u-\mathrm{i} k u=o\left(\|x\|^{\frac{1-n}{2}}\right) \quad \text { as }\|x\| \rightarrow \infty,
\end{aligned}
$$

where $D$ is a bounded domain in $R^{n}(n=1,2,3), \partial_{r}$ is the radial derivative, and $k$ is the nondimensional wave number: $k=\frac{\omega L}{c}$, where $\omega$ is a given frequency, $L$ is the measure of the domain, and $c$ is the sound speed in the acoustic medium.

Problem (1.1) presents a great challenge to numerical analysts and computational scientists because (i) the domain is unbounded, and (ii) the solution is highly oscillatory (when $k$ is large) and decays slowly. There is abundant literature on different numerical techniques that have been developed for this problem, such as boundary element methods [5], infinite element methods [11], methods using nonreflecting boundary conditions [14], perfectly matched layers (PML) [2], among others. In many of these approaches, an essential step is to solve the following problem:

$$
\begin{aligned}
& -\Delta u-k^{2} u=f \quad \text { in } \Omega:=B \backslash D, \\
& \left.u\right|_{\partial D}=0,\left.\quad\left(\partial_{r} u-\mathrm{i} k u\right)\right|_{\partial B}=g,
\end{aligned}
$$

where $\partial_{r}$ is the outward normal derivative, $f, g$ are given data, and $B$ is a sufficiently large ball containing $D$.

The analysis and implementation of numerical schemes for (1.2) are challenging when the wave number $k$ is large. The Galerkin finite element method (FEM) for (1.2)

\footnotetext{
${ }^{*}$ Received by the editors April 26, 2004; accepted for publication (in revised form) October 11, 2004; published electronically June 30, 2005.

http://www.siam.org/journals/sinum/43-2/60733.html

${ }^{\dagger}$ Department of Mathematics, Purdue University, West Lafayette, IN 47907 (shen@math.purdue. edu, lwang@math.purdue.edu). The work of the first author was partially supported by NSF grant DMS-0311915.
} 
in the one-dimensional case was first carried out in [8], where the well-posedness and error estimates of the Galerkin FEM were established under the condition $k^{2} h \lesssim 1$ using the Green's function and an argument due to Schatz [21]. A refined analysis for (1.2) in the one-dimensional case was performed in [18] (resp., [19]) for the $h$ version (resp., $h p$ version) of FEM, where the well-posedness and error estimates were established under the condition $k h \lesssim 1$ using the discrete Green's functions. The proofs in these works rely heavily on the use of explicit forms of continuous and/or discrete Green's functions. Hence, it is extremely complicated, if not impossible, to extend to more general cases and higher space dimensions.

On the other hand, the error estimates in the aforementioned papers concluded that the mesh condition $k^{2} h \lesssim 1$ has to be verified for the error estimates to be independent of $k$. This so-called pollution effect associated with high wave numbers was discussed in detail in [1]. It is well known [13] that spectral methods are suitable for problems with highly oscillatory solutions since they require fewer grid points per wavelength compared with finite difference methods and FEMs. Furthermore, since the convergence rate of spectral methods increases with the smoothness of the solution, the effect of pollution on the convergence rate of spectral methods is much less significant for smooth (but highly oscillatory) solutions. Hence, it is advantageous to use a spectral method for the Helmholtz equation (1.2) with high wave numbers.

In a recent work [7], Cummings and Feng obtained sharp regularity results for (1.2) in general two- or three-dimensional domains by using Rellich identities instead of using representations in terms of double-layer potentials (cf. [10]). Their analysis not only leads to sharper regularity results but also greatly simplifies the usual process for obtaining a priori estimates and is applicable to general and multidimensional starshaped domains. Unfortunately, the technique used in [7] cannot be directly applied to Galerkin FEMs because the finite element subspaces do not contain the special test functions used in [7]. However, the situation is different in a spectral-Galerkin method, for which the procedure in [7] can be applied.

We consider in this paper the spectral-Galerkin method for the Helmholtz equation with high wave numbers. In the next section, we set up a prototypical onedimensional Helmholtz equation which is derived from a multidimensional Helmholtz equation, and we establish its well-posedness; then we derive a priori estimates which are essential for the error analysis. In section 3, we introduce the spectral-Galerkin method and use the same arguments for the space continuous problem to establish the well-posedness and a priori estimates for the discrete problem; then we employ some new optimal Jacobi approximation results to carry out a complete error analysis. In section 4 , we consider an alternative formulation which leads to an efficient numerical algorithm and present some illustrative numerical results. We extend our analysis to multidimensional domains in section 5 .

We now introduce some notation. Let $\omega(x)$ be a given real weight function in $I=(a, b)$, which is not necessary in $L^{1}(I)$. We denote by $L_{\omega}^{2}(I)$ a Hilbert space of real or complex functions with inner product and norm

$$
(u, v)_{\omega}=\int_{I} u(r) \overline{v(r)} \omega(r) d r, \quad\|u\|_{\omega}=(u, u)_{\omega}^{\frac{1}{2}},
$$

where $\bar{v}$ is the complex conjugate of $v$. Then the weighted Sobolev spaces $H_{\omega}^{s}(I)(s=$ $0,1,2, \ldots)$ can be defined as usual with inner products, norms, and seminorms denoted by $(\cdot, \cdot)_{s, \omega},\|\cdot\|_{s, \omega}$, and $|\cdot|_{s, \omega}$, respectively. For real $s>0, H_{\omega}^{s}(I)$ is defined by space interpolation. The subscript $\omega$ will be omitted from the notation in the case $\omega \equiv 1$. For simplicity, we denote $\partial_{r}^{l} v=\frac{d^{l} v}{d r^{l}}, l \geq 1$. 
2. Model equation and a priori estimates. Since a global spectral method is most efficient on regular domains, we shall restrict our attention to the following special cases $(b>a \geq 0)$ :

- One-dimensional case (1-D): $D=(0, a)$ and $B=(0, b)$.

- Two-dimensional case (2-D): $D=\left\{(x, y): x^{2}+y^{2}<a^{2}\right\}$ and $B=\{(x, y)$ : $\left.x^{2}+y^{2}<b^{2}\right\}$.

- Three-dimensional case (3-D): $D=\left\{(x, y, z): x^{2}+y^{2}+z^{2}<a^{2}\right\}$ and $B=$ $\left\{(x, y, z): x^{2}+y^{2}+z^{2}<b^{2}\right\}$.

In the 2-D (resp., 3-D) case, we expand functions in polar (resp., spherical) coordinates, i.e., $u=\sum u_{m}(r) e^{i m \theta}$ (resp., $u=\sum u_{l m}(r) Y_{l, m}(\theta, \phi)$, where $\left\{Y_{l, m}(\theta, \phi)\right\}$ are the usual spherical harmonic functions). Hence, the problem (1.2) reduces, after a polar (when $n=2$ ) or spherical (when $n=3$ ) transform, to a sequence (for each $m$ in 2-D and $(l, m)$ in 3-D) of 1-D equations (for brevity, we use $u$ to denote $u_{m} / u_{l m}$, and likewise for $f$ and $g$, below):

$$
-\frac{1}{r^{n-1}} \partial_{r}\left(r^{n-1} \partial_{r} u\right)+d_{m} \frac{u}{r^{2}}-k^{2} u=f, \quad r \in(a, b), n=1,2,3, m \geq 0
$$

$\left(d_{m}=0, m^{2}, m(m+1)\right.$ for $n=1,2,3$, respectively), with suitable boundary conditions to be specified below.

If $a>0$, the coefficients $r^{n-1}$ and $r^{-2}$ in (2.1) are uniformly bounded, so (2.1) with $a>0$ is easier to handle than the case $a=0$. Hence, for brevity of presentation, we shall be concerned mainly with the case $a=0$, while some results for $a>0$ will be stated without proof in section 5. On the other hand, the character of (2.1) does not change with the change of variable: $r \rightarrow r b$. Consequently, it suffices to consider the problem $(2.1)$ in $I:=(0,1)$. The appropriate boundary conditions for $(2.1)$ are the pole conditions at $r=0$,

$$
u(0)=0 \quad \text { if } n=1 \text { and if } n=2 \text { with } m>0,
$$

and the Robin boundary condition (derived from the Sommerfeld radiation boundary condition) at $r=1$,

$$
u^{\prime}(1)-\mathrm{i} k u(1)=g .
$$

We note that error estimates for finite element approximations to the Helmholtz equation (2.1) with high wave numbers were derived in $[8,18,19]$ for the 1-D case, and in $[9,6]$ for 2-D cases and in [12] for the 1-D Bessel equation reduced from a 3-D spherical domain, respectively.

Let $\mathbb{N}$ be the set of all nonnegative integers and let $P_{N}$ be the space of all polynomials of degree at most $N$. We shall use $c$ to denote a generic positive constant independent of any function, the wave frequency $k$, the radial/spherical frequency $m$, and the number of modes $N$. We use the expression $A \lesssim B$ to mean that there exists a generic positive constant $c$ such that $A \leq c B$.

2.1. Variational formulation and weak solution. Let us denote $\omega^{\alpha}(r)=r^{\alpha}$ and $\omega(r)=r$. We define a Hilbert space,

$$
X:=X(m, n):=\left\{u \in H_{\omega^{n-1}}^{1}(I): u \in L_{\omega^{n-3}}^{2}(I) \text { for } n=2,3 ; u \text { satisfies }(2.2)\right\},
$$

and a sesquilinear form on $X \times X$,

$$
\begin{aligned}
\mathcal{B}(u, v):=\mathcal{B}_{m n}(u, v) & :=\left(\partial_{r} u, \partial_{r} v\right)_{\omega^{n-1}}+d_{m}(u, v)_{\omega^{n-3}}-k^{2}(u, v)_{\omega^{n-1}} \\
& -\mathrm{i} k u(1) \overline{v(1)} .
\end{aligned}
$$


Note that to lighten the presentation, we will often omit $m$ and $n$ from the notation.

Then the weak formulation of (2.1)-(2.2) is to find $u \in X$ such that

$$
\mathcal{B}(u, v)=(f, v)_{\omega^{n-1}}+g \overline{v(1)} \forall v \in X, \quad n=1,2,3 .
$$

TheOREM 2.1. Given $f \in X^{\prime}$, the problem (2.5) admits a unique weak solution.

Proof. This result with $n=1$ was established in [8, 18]. Hence, we shall prove only the cases with $n=2$ and 3 .

We first consider the uniqueness. It suffices to show that $u=0$ is the only solution of the problem (2.5) with $f \equiv 0$ and $g=0$.

Taking $v=u$ in (2.5) with $f \equiv 0$ and $g=0$, we find from (2.4) that

$$
\mathcal{B}(u, u)=\left\|\partial_{r} u\right\|_{\omega^{n-1}}^{2}+d_{m}\|u\|_{\omega^{n-3}}^{2}-k^{2}\|u\|_{\omega^{n-1}}^{2}-\mathrm{i} k|u(1)|^{2}=0,
$$

which implies immediately $u(1)=0$.

Next, let $J_{\mu}(r)$ be the Bessel function of the first kind of order $\mu$. We recall that

$$
\phi_{m}(r ; h, n):= \begin{cases}J_{m}(h r), & n=2, r, h>0, \\ \frac{1}{\sqrt{r}} J_{m+\frac{1}{2}}(h r), & n=3, r, h>0,\end{cases}
$$

is the solution of the modified Bessel equation (cf. [25]):

$$
-\frac{1}{r^{n-1}} \partial_{r}\left(r^{n-1} \partial_{r} \phi_{m}\right)-\left(h^{2}-\frac{d_{m}}{r^{2}}\right) \phi_{m}=0, \quad n=2,3, m \geq 0 .
$$

Let $\left\{\xi_{j}\right\}_{j=1}^{\infty}$ be the set of all positive real zeros of the Bessel function $J_{m+\frac{n}{2}-1}(r)$. Then $\left\{\phi_{m}\left(r ; \xi_{j}, n\right)\right\}_{j=1}^{\infty}$ forms a complete orthogonal system in $L_{\omega^{n-1}}^{2}(I)$ (cf. [26]), namely,

$$
\begin{aligned}
& \int_{0}^{1} \phi_{m}\left(r ; \xi_{j}, n\right) \phi_{m}\left(r ; \xi_{l}, n\right) r^{n-1} d r \\
& =\int_{0}^{1} J_{m+\frac{n}{2}-1}\left(r \xi_{j}\right) J_{m+\frac{n}{2}-1}\left(r \xi_{l}\right) r d r=\frac{1}{2} J_{m+\frac{n}{2}}^{2}\left(\xi_{j}\right) \delta_{j, l} .
\end{aligned}
$$

Since $u \in L_{\omega^{n-1}}^{2}(I)$, we can write

$$
u(r)=\sum_{j=1}^{\infty} \tilde{u}_{m}^{(j)} \phi_{m}\left(r ; \xi_{j}, n\right)
$$

with

$$
\tilde{u}_{m}^{(j)}=\frac{1}{\gamma_{m}^{(j)}} \int_{0}^{1} u(r) \phi_{m}\left(r ; \xi_{j}, n\right) r^{n-1} d r, \quad \gamma_{m}^{(j)}=\frac{1}{2} J_{m+\frac{n}{2}}^{2}\left(\xi_{j}\right) .
$$

Thanks to $u(1)=0$, we derive from (2.8) with $h=\xi_{j},(2.11)$, and integration by parts that

$$
\begin{aligned}
0=\mathcal{B}\left(u, \phi_{m}\right) & =\int_{0}^{1} u(r)\left\{-\frac{1}{r^{n-1}} \partial_{r}\left(r^{n-1} \partial_{r} \phi_{m}\right)-\left(k^{2}-\frac{d_{m}}{r^{2}}\right) \phi_{m}\right\} r^{n-1} d r \\
& =\left(\xi_{j}^{2}-k^{2}\right) \int_{0}^{1} u(r) \phi_{m}\left(r ; \xi_{j}, n\right) r^{n-1} d r=\left(\xi_{j}^{2}-k^{2}\right) \gamma_{m}^{(j)} \tilde{u}_{m}^{(j)}
\end{aligned}
$$


If $J_{m+\frac{n}{2}-1}(k) \neq 0$ (i.e., $k \neq \xi_{j}$ for all $j \geq 1$ ), then (2.12) implies $\tilde{u}_{m}^{(j)}=0$ for all $j$. Accordingly, we have $u \equiv 0$ (cf. (2.10)).

On the other hand, if $J_{m+\frac{n}{2}-1}(k)=0$, then $k=\xi_{j_{0}}$ for some $j_{0} \geq 1$. We then derive from $(2.12)$ that $\tilde{u}_{m}^{(j)}=0$ for all $j \neq j_{0}$. Thus, by (2.10),

$$
u(r)=\tilde{u}_{m}^{\left(j_{0}\right)} \phi_{m}\left(r ; \xi_{j_{0}}, n\right),
$$

and it remains to verify $\tilde{u}_{m}^{\left(j_{0}\right)}=0$. Due to $u(1)=0$, integration by parts yields

$$
\int_{0}^{1} \partial_{r} u(r) \partial_{r}\left(r^{m}\right) r^{n-1} d r=-d_{m} \int_{0}^{1} u(r) r^{m+n-3} d r, \quad n=2,3, m \geq 0 .
$$

Taking $v=r^{m}(\in X)$ in (2.5), we obtain from (2.13) that

$$
\begin{aligned}
0 & =\mathcal{B}\left(u, r^{m}\right)=\tilde{u}_{m}^{\left(j_{0}\right)} \mathcal{B}\left(\phi_{m}\left(\cdot ; \xi_{j_{0}}, n\right), r^{m}\right) \\
& =-k^{2} \tilde{u}_{m}^{\left(j_{0}\right)} \int_{0}^{1} \phi_{m}\left(r ; \xi_{j_{0}}, n\right) r^{m+n-1} d r=-k^{2} \tilde{u}_{m}^{\left(j_{0}\right)} \int_{0}^{1} J_{m+\frac{n}{2}-1}\left(r \xi_{j_{0}}\right) r^{m+\frac{n}{2}} d r .
\end{aligned}
$$

We recall that $r^{\mu}, \mu \geq 0$, can be expanded as (see [26, p. 581])

$$
r^{\mu}=\sum_{j=1}^{\infty} \frac{2 J_{\mu}\left(r \xi_{j}\right)}{\xi_{j} J_{\mu+1}\left(\xi_{j}\right)}, \quad 0 \leq r<1 .
$$

Inserting (2.16) with $\mu=m+\frac{n}{2}-1$ into (2.15) and using the orthogonality (2.9) lead to

$$
0=-k^{2} \tilde{u}_{m}^{\left(j_{0}\right)} \int_{0}^{1} J_{m+\frac{n}{2}-1}\left(r \xi_{j_{0}}\right) r^{m+\frac{n}{2}} d r=-k^{2} \tilde{u}_{m}^{\left(j_{0}\right)} \frac{J_{m+\frac{n}{2}}\left(\xi_{j_{0}}\right)}{\xi_{j_{0}}} .
$$

This implies $\widetilde{u}_{m}^{\left(j_{0}\right)}=0$. Hence, we have $u \equiv 0$, which implies the uniqueness.

To prove the existence, we note from (2.6) that the following Gårding-type inequality holds:

$$
\operatorname{Re}(\mathcal{B}(u, u)) \geq\left\|\partial_{r} u\right\|_{\omega^{n-1}}^{2}+d_{m}\|u\|_{\omega^{n-3}}^{2}-k^{2}\|u\|_{\omega^{n-1}}^{2} .
$$

Since all the arguments above apply also to the dual problem of (2.5), by the classical Fredholm alternative argument (see, for instance, [20, p. 194]); problem (2.5) either has a nontrivial solution with $f \equiv 0$ and $g=0$ or it has at least one solution for every $f \in X^{\prime}$. Since the uniqueness is proved, existence follows from the above argument.

\subsection{A priori estimates.}

THEOREM 2.2. If $f \in L_{\omega^{n-1}}^{2}(I)$, we have

$$
\left\|\partial_{r} u\right\|_{\omega^{n-1}}+\sqrt{d_{m}}\|u\|_{\omega^{n-3}}+k\|u\|_{\omega^{n-1}} \lesssim|g|+\|f\|_{\omega^{n-1}}, \quad n=1,2,3 .
$$

Proof. The proof consists of taking two different test functions in (2.5). The first test function is the usual one. As in [7], the key step is to choose a suitable second test function which enables us to obtain a priori estimates without using the Green's functions as in $[8,18,19]$. In the following proof, $\varepsilon_{j}>0,1 \leq j \leq 5$, are adjustable real numbers. 
Step 1 . We take $v=u$ in (2.5) whose imaginary and real parts are as follows:

$$
\begin{aligned}
& -k|u(1)|^{2}=\operatorname{Im}(g \overline{u(1)})+\operatorname{Im}(f, u)_{\omega^{n-1}}, \\
& \left\|\partial_{r} u\right\|_{\omega^{n-1}}^{2}+d_{m}\|u\|_{\omega^{n-3}}^{2}-k^{2}\|u\|_{\omega^{n-1}}^{2}=\operatorname{Re}(g \overline{u(1)})+\operatorname{Re}(f, u)_{\omega^{n-1}} .
\end{aligned}
$$

Applying the Cauchy-Schwarz inequality to the imaginary part, we obtain

$$
\begin{aligned}
k|u(1)|^{2} & \leq|\operatorname{Im}(g \overline{u(1)})|+\left|\operatorname{Im}(f, u)_{\omega^{n-1}}\right|, \\
& \leq \frac{k}{2}|u(1)|^{2}+\frac{1}{2 k}|g|^{2}+\frac{\varepsilon_{1} k}{2}\|u\|_{\omega^{n-1}}^{2}+\frac{1}{2 \varepsilon_{1} k}\|f\|_{\omega^{n-1}}^{2} ;
\end{aligned}
$$

likewise, we obtain from the real part that

$$
\begin{aligned}
& \left\|\partial_{r} u\right\|_{\omega^{n-1}}^{2}+d_{m}\|u\|_{\omega^{n-3}}^{2} \leq k^{2}\|u\|_{\omega^{n-1}}^{2}+|\operatorname{Re}(g \overline{u(1)})|+\left|\operatorname{Re}(f, u)_{\omega^{n-1}}\right| \\
& \quad \leq k^{2}\|u\|_{\omega^{n-1}}^{2}+\varepsilon_{2} k^{2}|u(1)|^{2}+\frac{1}{4 \varepsilon_{2} k^{2}}|g|^{2}+\frac{\varepsilon_{3} k^{2}}{2}\|u\|_{\omega^{n-1}}^{2}+\frac{1}{2 \varepsilon_{3} k^{2}}\|f\|_{\omega^{n-1}}^{2} .
\end{aligned}
$$

Therefore, by (2.20),

$$
|u(1)|^{2} \leq \varepsilon_{1}\|u\|_{\omega^{n-1}}^{2}+\frac{1}{k^{2}}|g|^{2}+\frac{1}{\varepsilon_{1} k^{2}}\|f\|_{\omega^{n-1}}^{2},
$$

and by (2.21)-(2.22) with $\varepsilon_{2}=\frac{\varepsilon_{3}}{2 \varepsilon_{1}}$,

$$
\begin{aligned}
\left\|\partial_{r} u\right\|_{\omega^{n-1}}^{2}+ & d_{m}\|u\|_{\omega^{n-3}}^{2} \leq\left(1+\varepsilon_{3}\right) k^{2}\|u\|_{\omega^{n-1}}^{2} \\
& +\left(\frac{\varepsilon_{3}}{2 \varepsilon_{1}}+\frac{\varepsilon_{1}}{2 \varepsilon_{3} k^{2}}\right)|g|^{2}+\left(\frac{\varepsilon_{3}}{2 \varepsilon_{1}^{2}}+\frac{1}{2 \varepsilon_{3} k^{2}}\right)\|f\|_{\omega^{n-1}}^{2} .
\end{aligned}
$$

It remains to bound $k^{2}\|u\|_{\omega^{n-1}}^{2}$.

Step 2. Using a usual regularity argument, one can easily derive that, for $f \in$ $L_{\omega^{n-1}}^{2}(I)$, the weak solution of (2.5) satisfies $r \partial_{r} u \in X$, and we now consider the real part of (2.5) with $v=2 r \partial_{r} u$. After integrating by parts, the first three terms become

$$
\begin{gathered}
2 \operatorname{Re}\left(\partial_{r} u, \partial_{r}\left(r \partial_{r} u\right)\right)_{\omega^{n-1}}=\left|\partial_{r} u(1)\right|^{2}+(2-n)\left\|\partial_{r} u\right\|_{\omega^{n-1}}^{2} \\
2 \operatorname{Re}\left(u, r \partial_{r} u\right)_{\omega^{n-3}}=|u(1)|^{2}+(2-n)\|u\|_{\omega^{n-3}}^{2} ; \\
-2 k^{2} \operatorname{Re}\left(u, r \partial_{r} u\right)_{\omega^{n-1}}=-k^{2}|u(1)|^{2}+n k^{2}\|u\|_{\omega^{n-1}}^{2} .
\end{gathered}
$$

Consequently, the real part of (2.5) with $v=2 r \partial_{r} u$ is

$$
\begin{gathered}
(2-n)\left(\left\|\partial_{r} u\right\|_{\omega^{n-1}}^{2}+d_{m}\|u\|_{\omega^{n-3}}^{2}\right)+n k^{2}\|u\|_{\omega^{n-1}}^{2}+\left|\partial_{r} u(1)\right|^{2}+d_{m}|u(1)|^{2} \\
=k^{2}|u(1)|^{2}+2 \operatorname{Re}\left((\mathrm{i} k u(1)+g) \overline{\partial_{r} u(1)}\right)+2 \operatorname{Re}\left(f, r \partial_{r} u\right)_{\omega^{n-1}}
\end{gathered}
$$

We now proceed separately for the three different cases.

Case (i): $n=1$. Thanks to $d_{m}=0$, we derive from (2.25) and the CauchySchwarz inequality that

$$
\begin{aligned}
\left\|\partial_{r} u\right\|^{2} & +k^{2}\|u\|^{2}+\left|\partial_{r} u(1)\right|^{2} \leq k^{2}|u(1)|^{2}+\frac{1}{2}\left|\partial_{r} u(1)\right|^{2} \\
& +2 k^{2}|u(1)|^{2}+2|g|^{2}+\frac{1}{2}\left\|\partial_{r} u\right\|^{2}+2\|f\|^{2} .
\end{aligned}
$$


Hence, we obtain from (2.22) that

$$
\begin{aligned}
\frac{1}{2}\left\|\partial_{r} u\right\|^{2} & +k^{2}\|u\|^{2}+\frac{1}{2}\left|\partial_{r} u(1)\right|^{2} \leq 3 \varepsilon_{1} k^{2}\|u\|^{2}+c\left(|g|^{2}+\left(\varepsilon_{1}^{-1}+2\right)\|f\|^{2}\right) \\
& \leq \frac{k^{2}}{2}\|u\|^{2}+c\left(|g|^{2}+\|f\|^{2}\right),
\end{aligned}
$$

where we have taken $\varepsilon_{1}=\frac{1}{6}$ to derive the last inequality. This implies (2.18) with $n=1$.

Case (ii): $n=2$. Similarly, we have from (2.22), (2.23), and (2.25) that

$$
\begin{aligned}
2 k^{2}\|u\|_{\omega}^{2} & +\left|\partial_{r} u(1)\right|^{2}+d_{m}|u(1)|^{2} \leq \frac{1}{2}\left|\partial_{r} u(1)\right|^{2}+3 k^{2}|u(1)|^{2} \\
& +2|g|^{2}+\varepsilon_{4}\left\|\partial_{r} u\right\|_{\omega}^{2}+\varepsilon_{4}^{-1}\|f\|_{\omega}^{2} \\
& \leq \frac{1}{2}\left|\partial_{r} u(1)\right|^{2}+\left(3 \varepsilon_{1}+\varepsilon_{4}\left(1+\varepsilon_{3}\right)\right) k^{2}\|u\|_{\omega}^{2}+C_{1}|g|^{2}+C_{2}\|f\|_{\omega}^{2},
\end{aligned}
$$

where $C_{1}$ and $C_{2}$ are two positive constants in terms of $\varepsilon_{1}, \varepsilon_{3}$, and $\varepsilon_{4}$. We take $\varepsilon_{1}=1 / 6, \varepsilon_{3}=1, \varepsilon_{4}=1 / 4$ and obtain that

$$
k^{2}\|u\|_{\omega}^{2}+d_{m}|u(1)|^{2}+\frac{1}{2}\left|\partial_{r} u(1)\right|^{2} \lesssim|g|^{2}+\|f\|_{\omega}^{2} .
$$

A combination of (2.23) and (2.28) leads to (2.18) with $n=2$.

Case (iii): $n=3$. As in the derivation of Case (ii), using (2.22), (2.23), and (2.25) yields

$$
\begin{gathered}
3 k^{2}\|u\|_{\omega^{2}}^{2}+\left|\partial_{r} u(1)\right|^{2}+d_{m}|u(1)|^{2} \leq\left\|\partial_{r} u\right\|_{\omega^{2}}^{2}+d_{m}\|u\|^{2}+\frac{1}{2}\left|\partial_{r} u(1)\right|^{2}+3 k^{2}|u(1)|^{2} \\
+2|g|^{2}+\varepsilon_{5}\left\|\partial_{r} u\right\|_{\omega^{2}}^{2}+\varepsilon_{5}^{-1}\|f\|_{\omega^{2}}^{2} \\
\leq \frac{1}{2}\left|\partial_{r} u(1)\right|^{2}+\left(3 \varepsilon_{1}+\left(1+\varepsilon_{5}\right)\left(1+\varepsilon_{3}\right)\right) k^{2}\|u\|_{\omega^{2}}^{2}+C_{3}|g|^{2}+C_{4}\|f\|_{\omega^{2}}^{2}
\end{gathered}
$$

where $C_{3}$ and $C_{4}$ are two positive constants depending only on $\varepsilon_{1}, \varepsilon_{3}$, and $\varepsilon_{5}$. Taking $\varepsilon_{1}=2 / 27$ and $\varepsilon_{3}=\varepsilon_{5}=1 / 3$ such that $3 \varepsilon_{1}+\left(1+\varepsilon_{5}\right)\left(1+\varepsilon_{3}\right)=2$ gives

$$
k^{2}\|u\|_{\omega^{2}}^{2}+d_{m}|u(1)|^{2}+\frac{1}{2}\left|\partial_{r} u(1)\right|^{2} \lesssim|g|^{2}+\|f\|_{\omega^{2}}^{2} .
$$

This completes the proof.

Remark 2.1. We have also proved that

$$
\left|\partial_{r} u(1)\right|+\sqrt{d_{m}}|u(1)|+k|u(1)| \lesssim|g|+\|f\|_{\omega^{n-1}}, \quad n=1,2,3 .
$$

Remark 2.2. A combination of (2.1) and (2.18) leads to

$$
\begin{gathered}
|u|_{2} \lesssim k|g|+(1+k)\|f\| \quad \text { if } n=1, \\
\left\|D^{2} u\right\| \lesssim k|g|+(1+k)\|f\|_{\omega^{n-1}} \quad \text { if } n=2,3,
\end{gathered}
$$

where $D^{2} u=-\partial_{r}\left(r^{n-1} \partial_{r} u\right)+d_{m} r^{n-3} u$.

3. Spectral-Galerkin approximation. In this section, we shall present the spectral-Galerkin scheme and analyze its errors in suitably weighted Sobolev spaces. 
3.1. Spectral-Galerkin solution. Let us denote $X_{N}:=X \cap P_{N}$, where $P_{N}$ is the space of all polynomials of degree at most $N$. The spectral-Galerkin approximation of (2.5) is to find $u_{N} \in X_{N}$ such that

$$
\mathcal{B}\left(u_{N}, v_{N}\right)=\left(f, v_{N}\right)_{\omega^{n-1}}+g \overline{v_{N}(1)} \quad \forall v_{N} \in X_{N} .
$$

We observe that the sesquilinear form $\mathcal{B}(\cdot, \cdot)$ is not coercive in $X_{N} \times X_{N}$. To prove the well-posedness of (3.1) with $n=1$, Douglas et al. [8] used an argument due to Schatz [21] for the (finite element) discrete system under the condition $k^{2} h \lesssim 1$, while Ihlenburg and Babuška [18] used an inf-sup argument due to Babuška and Brezzi under the condition $k h \lesssim 1$. However, the spectral-Galerkin approximation space $X_{N}$, unlike in the Galerkin FEM, has the following property: For $u_{N} \in X_{N}$, we have $r \partial_{r} u_{N} \in X_{N}$. Hence, the proof of Theorem 2.2 is also valid for the discrete system (3.1); i.e., we have the following.

THEOREM 3.1. Let $u_{N}$ be a solution of (3.1). Then Theorem 2.2 holds with $u_{N}$ in place of $u$.

An immediate consequence is the following.

Corollary 3.1. The problem (3.1) admits a unique solution.

Proof. Since (3.1) is a finite-dimensional linear system, it suffices to prove the uniqueness. Now, let $u_{N}$ be a solution of (3.1) with $f \equiv 0$ and $g=0$. We derive from Theorem 3.1 that $u_{N} \equiv 0$, which implies the uniqueness.

Remark 3.1. It is interesting to note that while the existence of a solution for finite element approximations to the Helmholtz equation is guaranteed only under a mesh condition $k h \lesssim 1$ (see, for instance, $[8,18]$ ), the spectral-Galerkin approximation (3.1) always admits a unique solution, just as (2.1) itself.

3.2. Error estimates. Thanks to Theorems 2.2 and 3.1 , we can analyze the errors of the proposed scheme by comparing the numerical solution with some orthogonal projection of the exact solution as usual. For this purpose, let $\Pi_{N, n}^{1, m}: X \rightarrow X_{N}$ be an orthogonal projection, defined by

$$
\left(\partial_{r}\left(u-\Pi_{N, n}^{1, m} u\right), \partial_{r} v_{N}\right)_{\omega^{n-1}}=0 \quad \forall v_{N} \in X_{N}, \quad n=1,3 \quad \forall m \text { and } n=2 \text { with } m=0 .
$$

In order to estimate the errors between $u$ and $u_{N}$, we have to analyze the approximation properties of the projector $\Pi_{N, n}^{1, m}$ for functions in the following suitably weighted Sobolev spaces:

$$
\widetilde{H}_{\omega^{n-1}}^{s}(I):=\left\{u: u \in L_{\omega^{n-1}}^{2}(I),\left(r-r^{2}\right)^{\frac{k-1}{2}} \partial_{r}^{k} u \in L_{\omega^{n-1}}^{2}(I), 1 \leq k \leq s\right\},
$$

with the norm and seminorm

$$
\begin{aligned}
& \|u\|_{\widetilde{H}_{\omega^{n-1}}^{s}}=\left(\|u\|_{\omega^{n-1}}^{2}+\sum_{k=1}^{s}\left\|\left(r-r^{2}\right)^{\frac{k-1}{2}} \partial_{r}^{k} u\right\|_{\omega^{n-1}}^{2}\right)^{\frac{1}{2}}, \\
& |u|_{\widetilde{H}_{\omega^{n-1}}^{s}}=\left\|\left(r-r^{2}\right)^{\frac{s-1}{2}} \partial_{r}^{s} u\right\|_{\omega^{n-1}}, \quad s \geq 1, s \in \mathbb{N} .
\end{aligned}
$$

Lemma 3.1. For any $u \in X \cap \widetilde{H}_{\omega^{n-1}}^{s}(I)$, with $s \geq 1$ and $s \in \mathbb{N}$,

$$
\begin{array}{r}
\left\|\Pi_{N, n}^{1, m} u-u\right\|_{\mu, \omega^{n-1}} \lesssim N^{\mu-s}\left\|\left(r-r^{2}\right)^{\frac{s-1}{2}} \partial_{r}^{s} u\right\|_{\omega^{n-1}}, \\
\mu=0,1, \quad n=1,3 \quad \forall m \text { and } n=2 \text { with } m=0 .
\end{array}
$$


Proof. This result for $n=1$ can be derived from [4] with an improvement of the norm in terms of the weights $\left(r-r^{2}\right)^{\frac{s-1}{2}}$ given in [16]. For $n=2$ with $m=0$ and $n=3$, one can refer to $[15,16]$ for the proofs.

Next, we shall estimate $e_{N}=u_{N}-\Pi_{N, n}^{1, m} u$. We denote $\tilde{e}_{N}=u-\Pi_{N, n}^{1, m} u$.

LEMMA 3.2. Let $u$ and $u_{N}$ be, respectively, the solutions of (2.5) and (3.1). Then we have, for $n=1,3$ for all $m$ and $n=2$ with $m=0$,

$$
\begin{aligned}
& \left\|\partial_{r} e_{N}\right\|_{\omega^{n-1}}+\sqrt{d_{m}}\left\|e_{N}\right\|_{\omega^{n-3}}+k\left\|e_{N}\right\|_{\omega^{n-1}} \\
& \quad \lesssim \sqrt{d_{m}}\left(\left\|\partial_{r} \tilde{e}_{N}\right\|_{\omega^{n-1}}+\left\|\tilde{e}_{N}\right\|_{\omega^{n-3}}\right)+k^{2}\left\|\tilde{e}_{N}\right\|_{\omega^{n-1}}+k\left(1+d_{m} k^{-2}\right)\left|\tilde{e}_{N}(1)\right| .
\end{aligned}
$$

Proof. By (2.5) and (3.1), we have $\mathcal{B}\left(u-u_{N}, v_{N}\right)=0$ for all $v_{N} \in X_{N}$. Hence, we derive from (2.5) and (3.2) that, for any $v_{N} \in X_{N}$,

$$
\begin{aligned}
\mathcal{B}\left(e_{N}, v_{N}\right) & =\mathcal{B}\left(u-\Pi_{N, n}^{1, m} u, v_{N}\right) \\
& =d_{m}\left(\tilde{e}_{N}, v_{N}\right)_{\omega^{n-3}}-k^{2}\left(\tilde{e}_{N}, v_{N}\right)_{\omega^{n-1}}-\mathrm{i} k \tilde{e}_{N}(1) \overline{v_{N}(1)} .
\end{aligned}
$$

We can view (3.5) in the form of (2.5) with $u=e_{N}, g=-\mathrm{i} k \tilde{e}_{N}(1), f=-k^{2} \tilde{e}_{N}$ plus an extra term $d_{m}\left(\tilde{e}_{N}, v_{N}\right)_{\omega^{n-3}}$. Hence, as in the proof of Theorem 2.2, we take two different test functions $v_{N}=e_{N}, r \partial_{r} e_{N} \in X_{N}$ and estimate the extra term by

$$
\begin{aligned}
d_{m}\left|\left(\tilde{e}_{N}, e_{N}\right)_{\omega^{n-3}}\right| \leq & \varepsilon_{6} d_{m}\left\|e_{N}\right\|_{\omega^{n-3}}^{2}+\frac{d_{m}}{4 \varepsilon_{6}}\left\|\tilde{e}_{N}\right\|_{\omega^{n-3}}^{2}, \\
d_{m}\left|\left(\tilde{e}_{N}, r \partial_{r} e_{N}\right)_{\omega^{n-3}}\right|= & d_{m}\left|\tilde{e}_{N}(1) \overline{e_{N}(1)}-\left(\partial_{r} \tilde{e}_{N}, e_{N}\right)_{\omega^{n-2}}-(n-2)\left(\tilde{e}_{N}, e_{N}\right)_{\omega^{n-3}}\right| \\
\leq & \varepsilon_{7} k^{2}\left|e_{N}(1)\right|^{2}+\frac{d_{m}^{2}}{4 k^{2} \varepsilon_{7}}\left|\tilde{e}_{N}(1)\right|^{2}+\varepsilon_{8} d_{m}\left\|e_{N}\right\|_{\omega^{n-3}}^{2} \\
& +\frac{c d_{m}}{4 \varepsilon_{8}}\left(\left\|\partial_{r} \tilde{e}_{N}\right\|_{\omega^{n-1}}^{2}+\left\|\tilde{e}_{N}\right\|_{\omega^{n-3}}^{2}\right) .
\end{aligned}
$$

Thus, choosing suitable constants $\left\{\varepsilon_{j}\right\}_{j=6}^{8}$, and following a procedure similar to the proof of Theorem 2.2, we can derive

$$
\begin{aligned}
& \left\|\partial_{r} e_{N}\right\|_{\omega^{n-1}}^{2}+d_{m}\left\|e_{N}\right\|_{\omega^{n-3}}^{2}+k^{2}\left\|e_{N}\right\|_{\omega^{n-1}}^{2} \\
& \quad \lesssim d_{m}\left(\left\|\partial_{r} \tilde{e}_{N}\right\|_{\omega^{n-1}}^{2}+\left\|\tilde{e}_{N}\right\|_{\omega^{n-3}}^{2}\right)+k^{4}\left\|\tilde{e}_{N}\right\|_{\omega^{n-1}}^{2}+k^{2}\left(1+d_{m}^{2} k^{-4}\right)\left|\tilde{e}_{N}(1)\right|^{2},
\end{aligned}
$$

which leads to the desired result.

We now recall the following inequalities.

LEMMA 3.3.

$$
\begin{gathered}
|u(1)| \lesssim\|u\|_{\omega^{n-1}}^{\frac{1}{2}}\|u\|_{1, \omega^{n-1}}^{\frac{1}{2}} \quad \forall u \in H_{\omega^{n-1}}^{1}(I), \quad n=1,2,3, \\
\|u\| \lesssim\|u\|_{1, \omega^{2}} \quad \forall u \in H_{\omega^{2}}^{1}(I) .
\end{gathered}
$$

Proof. By the Sobolev inequality (see the appendix in [4]),

$$
|u(1)| \lesssim\|u\|_{L^{2}(1 / 2,1)}^{\frac{1}{2}}\|u\|_{H^{1}(1 / 2,1)}^{\frac{1}{2}} \lesssim\|u\|_{L_{\omega^{n-1}}^{2}(1 / 2,1)}^{\frac{1}{2}}\|u\|_{H_{\omega^{n-1}}^{1}(1 / 2,1)}^{\frac{1}{2}} \lesssim\|u\|_{\omega^{n-1}}^{\frac{1}{2}}\|u\|_{1, \omega^{n-1}}^{\frac{1}{2}} .
$$

Here, we used the fact that the weight function $r^{n-1}$ is uniformly bounded on $[1 / 2,1]$. The inequality (3.7b) follows directly from formula (13.5) of [3]. 
As a consequence of (3.7b) and Lemma 3.1, we derive that for $n=3$,

$$
\left\|\Pi_{N, n}^{1, m} u-u\right\|_{\omega^{n-3}} \lesssim\left\|\Pi_{N, n}^{1, m} u-u\right\|_{1, \omega^{n-1}} \lesssim N^{1-s}\left\|\left(r-r^{2}\right)^{\frac{s-1}{2}} \partial_{r}^{s} u\right\|_{\omega^{n-1}} .
$$

With the above preparations, we can now prove our main results.

THEOREM 3.2. Let $u$ and $u_{N}$ be, respectively, the solutions of (2.5) and (3.1) such that $u \in X \cap \widetilde{H}_{\omega^{n-1}}^{s}(I)$ with $s \geq 1, s \in \mathbb{N}$.

(i) For $n=1$ or $n=2,3, m=0$,

$$
\left\|\partial_{r}\left(u-u_{N}\right)\right\|_{\omega^{n-1}}+k\left\|u-u_{N}\right\|_{\omega^{n-1}} \lesssim\left(1+k^{2} N^{-1}\right) N^{1-s}\left\|\left(r-r^{2}\right)^{\frac{s-1}{2}} \partial_{r}^{s} u\right\|_{\omega^{n-1}} .
$$

(ii) For $n=3$ and $m>0$,

$$
\begin{aligned}
& \left\|\partial_{r}\left(u-u_{N}\right)\right\|_{\omega^{2}}+\sqrt{d_{m}}\left\|u-u_{N}\right\|+k\left\|u-u_{N}\right\|_{\omega^{2}} \\
& \quad \lesssim\left(\sqrt{d_{m}}+d_{m}^{2} k^{-4}+k^{2} N^{-1}\right) N^{1-s}\left\|\left(r-r^{2}\right)^{\frac{s-1}{2}} \partial_{r}^{s} u\right\|_{\omega^{2}},
\end{aligned}
$$

where $d_{m}=m(m+1)$.

Proof. We first prove (3.9). Since

$$
\begin{aligned}
\left\|\partial_{r}\left(u-u_{N}\right)\right\|_{\omega^{n-1}} & +k\left\|u-u_{N}\right\|_{\omega^{n-1}} \lesssim\left\|\partial_{r}\left(\Pi_{N, n}^{1, m} u-u\right)\right\|_{\omega^{n-1}} \\
& +k\left\|\Pi_{N, n}^{1, m} u-u\right\|_{\omega^{n-1}}+\left\|\partial_{r} e_{N}\right\|_{\omega^{n-1}}+k\left\|e_{N}\right\|_{\omega^{n-1}},
\end{aligned}
$$

formula (3.9) follows from Lemmas 3.1 and 3.2 and (3.7a).

Similarly, for $n=3$ and $m>0$, we derive from (3.7a) and Lemmas 3.1 and 3.2 that

$$
\begin{aligned}
\| \partial_{r}(u- & \left.u_{N}\right)\left\|_{\omega^{n-1}}+\sqrt{d_{m}}\right\| u-u_{N}\left\|_{\omega^{n-3}}+k\right\| u-u_{N} \|_{\omega^{n-1}} \\
\lesssim & \sqrt{d_{m}}\left(\left\|\partial_{r}\left(\Pi_{N, n}^{1, m} u-u\right)\right\|_{\omega^{n-1}}+\left\|\Pi_{N, n}^{1, m} u-u\right\|_{\omega^{n-3}}\right) \\
& +k^{2}\left\|\Pi_{N, n}^{1, m} u-u\right\|_{\omega^{n-1}}+k\left(1+d_{m} k^{-2}\right)\left|\left(\Pi_{N, n}^{1, m} u-u\right)(1)\right| \\
\lesssim & \sqrt{d_{m}}\left(\left\|\partial_{r}\left(\Pi_{N, n}^{1, m} u-u\right)\right\|_{\omega^{n-1}}+\left\|\Pi_{N, n}^{1, m} u-u\right\|_{\omega^{n-3}}\right) \\
& +2 k^{2}\left\|\Pi_{N, n}^{1, m} u-u\right\|_{\omega^{n-1}}+\left(1+d_{m} k^{-2}\right)^{2}\left\|\Pi_{N, n}^{1, m} u-u\right\|_{1, \omega^{n-1}} \\
\lesssim & \left(\sqrt{d_{m}}+\left(1+d_{m} k^{-2}\right)^{2}+k^{2} N^{-1}\right) N^{1-s}\left\|\left(r-r^{2}\right)^{\frac{s-1}{2}} \partial_{r}^{s} u\right\|_{\omega^{n-1}} \\
& +\sqrt{d_{m}}\left\|\Pi_{N, n}^{1, m} u-u\right\|_{\omega^{n-3}} .
\end{aligned}
$$

Hence, we can obtain (3.10) by using (3.8) to estimate the last term in (3.11).

Remark 3.2. For $n=1$, an error estimate of the same order as in (3.9) was derived in [19] for the $h p$ FEM under the condition $k h \lesssim 1$. Our estimate is valid without any restriction on $k$ and $N$ and is bounded by a weaker weighted seminorm.

Although we believe that the estimate (3.10), modulo perhaps a logarithmic term, is also valid for the case $n=2$ with $m>0$, the above proof cannot be directly extended to this case due to a breakdown in the Hardy inequality (cf. [17]) as $\varepsilon \rightarrow 0$,

$$
\int_{0}^{1} \frac{u^{2}}{r^{2}} r^{1-\varepsilon} d r \leq \frac{4}{\varepsilon} \int_{0}^{1}\left(\partial_{r} u\right)^{2} r^{1-\varepsilon} d r
$$


which indicates that $\left\|\Pi_{N, n}^{1, m} u-u\right\|_{\omega^{-1}}$ in the last term of (3.11) cannot be bounded by $\left\|\partial_{r}\left(\Pi_{N, n}^{1, m} u-u\right)\right\|_{\omega}$.

Next, we perform the error estimate for the case $n=2$ with $m>0$ by using a different approach.

Let $a_{m}(u, v):=\left(\partial_{r} u, \partial_{r} v\right)_{\omega}+d_{m}(u, v)_{\omega^{-1}}$ and define the orthogonal projection $\pi_{N}^{1, m}: X \rightarrow X_{N}$ by

$$
a_{m}\left(\pi_{N}^{1, m} u-u, v_{N}\right)=0 \quad \forall v_{N} \in X_{N} .
$$

To analyze the approximation properties of the above projector, we first consider an auxiliary projection. Let $\hat{\omega}=r(1-r)$, let $P_{N}^{0}:=\left\{u \in P_{N}: u(0)=u(1)=0\right\}$, and let $\pi_{N}$ be the $L_{\hat{\omega}^{-1}}^{2}$-orthogonal projection onto $P_{N}^{0}$ defined by

$$
\left(\pi_{N} u-u, v_{N}\right)_{\hat{\omega}^{-1}}=0 \quad \forall v_{N} \in P_{N}^{0} .
$$

The following result can be derived directly from the generalized Jacobi approximation with parameters $\alpha=\beta=-1$ (cf. Theorem 3.1 of [24]).

Lemma 3.4. For any $u \in L_{\hat{\omega}^{-1}}^{2}(I) \cap \widetilde{H}^{s}(I)$ with $s \geq 1, s \in \mathbb{N}$,

$$
\left\|\partial_{r}\left(\pi_{N} u-u\right)\right\|+N\left\|\left(\pi_{N} u-u\right)\right\|_{\hat{\omega}^{-1}} \lesssim N^{1-s}\left\|\left(r-r^{2}\right)^{\frac{s-1}{2}} \partial_{r}^{s} u\right\| .
$$

CoRollary 3.2. There exists an operator $\pi_{N}^{1}: H^{1}(I) \rightarrow P_{N}$ such that $\left(\pi_{N}^{1} u\right)(r)=$ $u(r)$ for $r=0,1$ and for any $u \in \widetilde{H}^{s}(I)$, with $s \geq 1, s \in \mathbb{N}$,

$$
\left\|\partial_{r}\left(\pi_{N}^{1} u-u\right)\right\|+N\left\|\pi_{N}^{1} u-u\right\|_{\hat{\omega}^{-1}} \lesssim N^{1-s}\left\|\left(r-r^{2}\right)^{\frac{s-1}{2}} \partial_{r}^{s} u\right\| .
$$

Proof. Let $u_{*}(r)=(1-r) u(0)+r u(1) \in P_{1}$ for all $u \in H^{1}(I)$. By construction, we have $\left(u-u_{*}\right)(r)=0$ for $r=0,1$. Next, we derive from the Hardy inequality (cf. [17]) that

$$
\begin{aligned}
\left(\int_{0}^{1}\left(u-u_{*}\right)^{2}\left(r-r^{2}\right)^{-1} d r\right)^{\frac{1}{2}} & \lesssim\left(\int_{0}^{1}\left(\partial_{r}\left(u-u_{*}\right)\right)^{2} d r\right)^{\frac{1}{2}} \\
\lesssim\left\|\partial_{r} u\right\|+|u(1)-u(0)| & \lesssim\left\|\partial_{r} u\right\|+\int_{0}^{1}\left|\partial_{r} u\right| d r \lesssim\left\|\partial_{r} u\right\| .
\end{aligned}
$$

Hence, $u-u_{*} \in L_{\hat{\omega}^{-1}}^{2}(I)$ and we can define

$$
\pi_{N}^{1} u=\pi_{N}\left(u-u_{*}\right)+u_{*} \in P_{N} \quad \forall u \in H^{1}(I) .
$$

Clearly, $\left(\pi_{N}^{1} u\right)(r)=u(r)$ for $r=0,1$, and by Lemma 3.4 ,

$$
\left\|\partial_{r}\left(\pi_{N}^{1} u-u\right)\right\|+N\left\|\left(\pi_{N}^{1} u-u\right)\right\|_{\hat{\omega}^{-1}} \lesssim N^{1-s}\left\|\left(r-r^{2}\right)^{\frac{s-1}{2}} \partial_{r}^{s}\left(u-u_{*}\right)\right\| .
$$

Since $\partial_{r}^{s} u_{*} \equiv 0$ for $s \geq 2$, and $\partial_{r} u_{*}=u(1)-u(0)$, which implies that

$$
\left\|\partial_{r} u_{*}\right\|=|u(1)-u(0)| \lesssim\left\|\partial_{r} u\right\|,
$$

the desired result follows from (3.17).

Using the above corollary leads to the following lemma. 
Lemma 3.5. For any $u \in X \cap \widetilde{H}^{s}(I)$ with $s \geq 1, s \in \mathbb{N}$,

$$
\begin{aligned}
&\left\|\partial_{r}\left(\pi_{N}^{1, m} u-u\right)\right\|_{\omega}+\sqrt{d_{m}}\left\|\pi_{N}^{1, m} u-u\right\|_{\omega^{-1}} \\
& \lesssim\left(1+\sqrt{d_{m}} N^{-1}\right) N^{1-s}\left\|\left(r-r^{2}\right)^{\frac{s-1}{2}} \partial_{r}^{s} u\right\| ; \\
&\left\|\pi_{N}^{1, m} u-u\right\|_{\omega} \lesssim\left(d_{m}^{-\frac{1}{2}}+N^{-1}\right) N^{1-s}\left\|\left(r-r^{2}\right)^{\frac{s-1}{2}} \partial_{r}^{s} u\right\| .
\end{aligned}
$$

Proof. The definition (3.13) implies that for any $\phi \in X_{N}$,

$$
a_{m}\left(\pi_{N}^{1, m} u-u, \pi_{N}^{1, m} u-u\right) \leq a_{m}(\phi-u, \phi-u) .
$$

Taking $\phi=\pi_{N}^{1} u \in X_{N}$ in (3.19), we obtain from Corollary 3.2 that

$$
\begin{aligned}
\left\|\partial_{r}\left(\pi_{N}^{1, m} u-u\right)\right\|_{\omega} & +\sqrt{d_{m}}\left\|\pi_{N}^{1, m} u-u\right\|_{\omega^{-1}} \lesssim\left\|\partial_{r}\left(\pi_{N}^{1} u-u\right)\right\|+\sqrt{d_{m}}\left\|\pi_{N}^{1} u-u\right\|_{\hat{\omega}^{-1}} \\
& \lesssim\left(1+\sqrt{d_{m}} N^{-1}\right) N^{1-s}\left\|\left(r-r^{2}\right)^{\frac{s-1}{2}} \partial_{r}^{s} u\right\| .
\end{aligned}
$$

Since $\left\|\pi_{N}^{1, m} u-u\right\|_{\omega} \leq\left\|\pi_{N}^{1, m} u-u\right\|_{\omega^{-1}}$, (3.18b) follows from (3.18a).

We can now derive an error estimate for the case $n=2$ with $m>0$.

TheOREM 3.3. If $u \in X \cap \widetilde{H}^{s}(I)$, with $s \geq 1$ and $s \in \mathbb{N}$, we have

$$
\begin{aligned}
& \left\|\partial_{r}\left(u-u_{N}\right)\right\|_{\omega}+\sqrt{d_{m}}\left\|u-u_{N}\right\|_{\omega^{-1}}+k\left\|u-u_{N}\right\|_{\omega} \\
& \quad \lesssim\left(\left(1+\sqrt{d_{m}} N^{-1}+d_{m}^{2} k^{-4}\right)+k^{2}\left(d_{m}^{-\frac{1}{2}}+N^{-1}\right)\right) N^{1-s}\left\|\left(r-r^{2}\right)^{\frac{s-1}{2}} \partial_{r}^{s} u\right\| .
\end{aligned}
$$

Proof. Let us still denote $e_{N}=u_{N}-\pi_{N}^{1, m} u$ and $\tilde{e}_{N}=u-\pi_{N}^{1, m} u$. Due to (3.13), the error equation (3.5) becomes

$$
\mathcal{B}\left(e_{N}, v_{N}\right)=-k^{2}\left(\tilde{e}_{N}, v_{N}\right)_{\omega}-\mathrm{i} k \tilde{e}_{N}(1) \overline{v_{N}(1)} .
$$

Consequently, (3.6) is changed to

$$
\left\|\partial_{r} e_{N}\right\|_{\omega}^{2}+d_{m}\left\|e_{N}\right\|_{\omega^{-1}}^{2}+k^{2}\left\|e_{N}\right\|_{\omega}^{2} \lesssim k^{4}\left\|\tilde{e}_{N}\right\|_{\omega}^{2}+k^{2}\left(1+d_{m}^{2} k^{-4}\right)\left|\tilde{e}_{N}(1)\right|^{2} .
$$

Thus, following a procedure similar to that in the proof of Theorem 3.2, and thanks to Lemma 3.5, we can obtain (3.20).

4. An alternate formulation and its numerical implementation. In this section, we shall give an alternate formulation for problem (2.1)-(2.3), which is more suitable for implementation and also leads to a convergence rate similar to that of Theorem 3.2 .

4.1. The formulation. We make the transform

$$
u(r)=v(r) e^{\mathrm{i} k r}, \quad f(r)=h(r) e^{\mathrm{i} k r}, \quad r \in I,
$$

and we convert the problem (2.1)-(2.3) to

$$
\begin{aligned}
& -\frac{1}{r^{n-1}} \partial_{r}\left(r^{n-1} \partial_{r} v\right)+d_{m} \frac{v}{r^{2}}-\mathrm{i} k\left(2 \partial_{r} v+(n-1) \frac{v}{r}\right)=h, \\
& r \in I:=(0,1), n=1,2,3, m \geq 0,
\end{aligned}
$$

where $v$ satisfies the Dirichlet boundary condition (2.2) and the Neumann boundary condition:

$$
v^{\prime}(1)=\tilde{g}:=g e^{-\mathrm{i} k}
$$


Let the spaces $X$ and $X_{N}$ be the same as before. The weak formulation of (4.2) with (2.2) and (4.3) is to find $v \in X$ such that

$$
\begin{aligned}
\widetilde{\mathcal{B}}(v, w) & :=\left(\partial_{r} v, \partial_{r} w\right)_{\omega^{n-1}}+d_{m}(v, w)_{\omega^{n-3}}-2 \mathrm{i} k\left(\partial_{r} v, w\right)_{\omega^{n-1}} \\
& -(n-1) \mathrm{i} k(v, w)_{\omega^{n-2}}=(h, w)_{\omega^{n-1}}+\tilde{g} \overline{w(1)} \quad \forall w \in X .
\end{aligned}
$$

The well-posedness of this formulation is guaranteed by (4.1) and Theorem 2.1.

The spectral-Galerkin approximation to (4.4) is to seek $v_{N} \in X_{N}$ such that

$$
\widetilde{\mathcal{B}}_{N}\left(v_{N}, w_{N}\right)=\left(h, w_{N}\right)_{\omega^{n-1}}+\tilde{g} \overline{w_{N}(1)} \quad \forall w_{N} \in X_{N} .
$$

Using a procedure similar to the one used before, we can derive corresponding a priori estimates and error estimates. For simplicity, we consider the case $g=0$.

THEOREM 4.1. Let $v$ and $v_{N}$ be the solutions of (4.4) and (4.5) with $\tilde{g}=0$ and $h \in L_{\omega^{n-1}}^{2}(I)$. Then

$$
\begin{gathered}
\left\|\partial_{r} v\right\|_{\omega^{n-1}}+\sqrt{d_{m}}\|v\|_{\omega^{n-3}} \lesssim\|h\|_{\omega^{n-1}} \\
\left\|\partial_{r} v_{N}\right\|_{\omega^{n-1}}+\sqrt{d_{m}}\left\|v_{N}\right\|_{\omega^{n-3}} \lesssim\|h\|_{\omega^{n-1}}
\end{gathered}
$$

Proof. As in the proof of Theorem 2.2, we take two different test functions in (4.4). We first take $w=v$ in (4.4), whose real part is

$$
\left\|\partial_{r} v\right\|_{\omega^{n-1}}^{2}+d_{m}\|v\|_{\omega^{n-3}}^{2}+2 k \operatorname{Im}\left(\partial_{r} v, v\right)_{\omega^{n-1}}=\operatorname{Re}(h, v)_{\omega^{n-1}},
$$

and using integration by parts, its imaginary part becomes

$$
-2 k \operatorname{Re}\left(\partial_{r} v, v\right)_{\omega^{n-1}}-(n-1) k\|v\|_{\omega^{n-2}}^{2}=-k|v(1)|^{2}=\operatorname{Im}(h, v)_{\omega^{n-1}} .
$$

Here, in the derivation of (4.8) (likewise for (4.10) below), we have used the fact $\operatorname{Re}(\mathrm{i}(u, v))=-\operatorname{Im}(u, v)$.

Next, we take $w=2 r \partial_{r} v(\in X)$ in (4.4), and thanks to (2.24a)-(2.24b), its real part becomes

$$
\begin{aligned}
(2-n)\left(\left\|\partial_{r} v\right\|_{\omega^{n-1}}^{2}\right. & \left.+d_{m}\|v\|_{\omega^{n-3}}^{2}\right)+d_{m}|v(1)|^{2} \\
& +2(n-1) k \operatorname{Im}\left(v, \partial_{r} v\right)_{\omega^{n-1}}=2 \operatorname{Re}\left(h, r \partial_{r} v\right)_{\omega^{n-1}} .
\end{aligned}
$$

As a consequence of (4.10), we have that for $n=1$ (we recall that $d_{m}=0$ in this case),

$$
\left\|\partial_{r} v\right\|^{2} \leq 2\|h\|_{\omega^{2}}\left\|\partial_{r} v\right\| \leq 2\|h\|\left\|\partial_{r} v\right\|,
$$

which implies (4.6) with $n=1$.

It remains to prove (4.6) with $n=2,3$. Since $\partial_{r} v(1)=0$, it is easy to verify

$$
\operatorname{Im}\left(\partial_{r} v, v\right)_{\omega^{n-1}}=-\operatorname{Im}\left(v, \partial_{r} v\right)_{\omega^{n-1}} .
$$

Therefore, multiplying (4.8) by $n-1$ and adding the resulting equation to (4.10), we derive from the Cauchy-Schwarz inequality that

$$
\begin{aligned}
& \left\|\partial_{r} v\right\|_{\omega^{n-1}}^{2}+d_{m}\|v\|_{\omega^{n-3}}^{2}+d_{m}|v(1)|^{2}=(n-1) \operatorname{Re}(h, v)_{\omega^{n-1}} \\
& \quad+2 \operatorname{Re}\left(h, r \partial_{r} v\right)_{\omega^{n-1}} \leq 2\|h\|_{\omega^{n-1}}\|v\|_{\omega^{n-1}}+\frac{1}{4}\left\|\partial_{r} v\right\|_{\omega^{n-1}}^{2}+4\|h\|_{\omega^{n-1}}^{2} .
\end{aligned}
$$


Clearly, we have

$$
|v(1)|^{2}=\int_{0}^{1} \partial_{r}\left(|v(r)|^{2} r^{n}\right) d r=n \int_{0}^{1}|v(r)|^{2} r^{n-1} d r+2 \int_{0}^{1} \partial_{r} v(r) \overline{v(r)} r^{n} d r
$$

and by the Cauchy-Schwarz inequality,

$$
n\|v\|_{\omega^{n-1}}^{2} \leq|v(1)|^{2}+2\|v\|_{\omega^{n-1}}\left\|\partial_{r} v\right\|_{\omega^{n+1}} \leq|v(1)|^{2}+\frac{n}{2}\|v\|_{\omega^{n-1}}^{2}+\frac{2}{n}\left\|\partial_{r} v\right\|_{\omega^{n-1}}^{2},
$$

which together with (4.9) leads to

$$
\begin{aligned}
\|v\|_{\omega^{n-1}}^{2} & \leq \frac{2}{n}|v(1)|^{2}+\frac{4}{n^{2}}\left\|\partial_{r} v\right\|_{\omega^{n-1}}^{2} \leq \frac{2}{n k}\left|\operatorname{Im}(h, v)_{\omega^{n-1}}\right|+\frac{4}{n^{2}}\left\|\partial_{r} v\right\|_{\omega^{n-1}}^{2} \\
& \leq \frac{1}{2}\|v\|_{\omega^{n-1}}^{2}+\frac{2}{n^{2} k^{2}}\|h\|_{\omega^{n-1}}^{2}+\frac{4}{n^{2}}\left\|\partial_{r} v\right\|_{\omega^{n-1}}^{2} .
\end{aligned}
$$

As a result of (4.13) and (4.14), we obtain

$$
\begin{aligned}
\left\|\partial_{r} v\right\|_{\omega^{n-1}}^{2} & +d_{m}\|v\|_{\omega^{n-3}}^{2} \leq 2\|h\|_{\omega^{n-1}}\left(\frac{2}{n k}\|h\|_{\omega^{n-1}}+\frac{2 \sqrt{2}}{n}\left\|\partial_{r} v\right\|_{\omega^{n-1}}\right) \\
& +\frac{1}{4}\left\|\partial_{r} v\right\|_{\omega^{n-1}}^{2}+4\|h\|_{\omega^{n-1}}^{2} \leq \frac{1}{2}\left\|\partial_{r} v\right\|_{\omega^{n-1}}^{2}+\left(\frac{4}{n k}+\frac{32}{n^{2}}+4\right)\|h\|_{\omega^{n-1}}^{2} .
\end{aligned}
$$

This completes the proof of (4.6).

Since $r \partial_{r} v_{N} \in X_{N}$, we have the same results for the numerical solution $v_{N}$.

Thanks to the above theorem, we can derive the following convergence result by using an argument similar to the proof of Theorem 3.2.

THEOREM 4.2. Let $v$ and $v_{N}$ be, respectively, the solutions of (4.4) and (4.5) with $\tilde{g}=0$, and we have

(i) for $n=1,3$ or $n=2, m=0$, and $v \in X \cap \widetilde{H}_{\omega^{n-1}}^{s}(I)$ with $s \geq 1$ and $s \in \mathbb{N}$,

$$
\left\|\partial_{r}\left(v-v_{N}\right)\right\|_{\omega^{n-1}}+\sqrt{d_{m}}\left\|v-v_{N}\right\|_{\omega^{n-3}} \lesssim\left(k+\sqrt{d_{m}}\right) N^{1-s}\left\|\left(r-r^{2}\right)^{\frac{s-1}{2}} \partial_{r}^{s} v\right\|_{\omega^{n-1}} ;
$$

(ii) for $n=2, m>0$, and $v \in X \cap \widetilde{H}^{s}(I)$, with $s \geq 1$ and $s \in \mathbb{N}$,

$$
\begin{aligned}
\| \partial_{r}(v & \left.-v_{N}\right)\left\|_{\omega}+\sqrt{d_{m}}\right\| v-v_{N} \|_{\omega^{-1}} \\
& \lesssim\left(\left(1+\sqrt{d_{m}} N^{-1}\right)+k^{2}\left(d_{m}^{-\frac{1}{2}}+N^{-1}\right)\right) N^{1-s}\left\|\left(r-r^{2}\right)^{\frac{s-1}{2}} \partial_{r}^{s} v\right\| .
\end{aligned}
$$

Proof. Let $\Pi_{N, n}^{1, m}$ be the orthogonal projection defined in (3.2), and denote $e_{N}=$ $v_{N}-\Pi_{N, n}^{1, m} v$ and $\hat{e}_{N}=v-\Pi_{N, n}^{1, m} v$. Like (3.5), the error equation is

$$
\widetilde{\mathcal{B}}\left(e_{N}, w_{N}\right)=d_{m}\left(\hat{e}_{N}, w_{N}\right)_{\omega^{n-3}}-2 \mathrm{i} k\left(\partial_{r} \hat{e}_{N}, w_{N}\right)_{\omega^{n-1}}-(n-1) \mathrm{i} k\left(\hat{e}_{N}, w_{N}\right)_{\omega^{n-2}} .
$$

Therefore, taking the test function $w_{N}=e_{N}, r \partial_{r} e_{N}$, setting $h=-2 \mathrm{i} k \partial_{r} \hat{e}_{N}-(n-$ 1)i $k r^{-1} \hat{e}_{N}$ in (4.5), and dealing with the term $d_{m}\left(\hat{e}_{N}, w_{N}\right)_{\omega^{n-3}}$ the same as that in the proof of Lemma 3.2, we obtain

$$
\left\|\partial_{r} e_{N}\right\|_{\omega^{n-1}}^{2}+d_{m}\left\|e_{N}\right\|_{\omega^{n-3}}^{2} \lesssim\left(k^{2}+d_{m}\right)\left(\left\|\partial_{r} \hat{e}_{N}\right\|_{\omega^{n-1}}^{2}+\left\|\hat{e}_{N}\right\|_{\omega^{n-3}}^{2}\right) .
$$

The rest of the proof of (4.15) is similar to that of Theorem 3.2.

The estimate (4.16) can be proved in the same fashion by using the results in Lemma 3.5. 


\subsection{Numerical implementations.}

4.2.1. Choice of basis functions. Without loss of generality, we still assume that $\tilde{g}=0$ in (4.2). For computational convenience, we transform $I=(0,1)$ to the reference interval $\hat{I}=(-1,1)$ with $x=2 r-1, r=\frac{1}{2}(1+x), r \in I, x \in \hat{I}$. As demonstrated in $[22,23]$, it is advantageous to construct basis function satisfying the underlying homogeneous boundary conditions by using compact combinations of orthogonal polynomials. Hence, we define

$W_{N}=W_{N}^{(m, n)}:=\left\{w \in P_{N}: w^{\prime}(1)=0 ; w(-1)=0\right.$ if $n=1$ and if $n=2$ with $\left.m>0\right\}$, and we let $L_{l}(x)$ denote the Legendre polynomial of degree $l$. Define

$$
\begin{aligned}
& \phi_{j}(x):=\left(L_{j}(x)+L_{j+1}(x)\right)-\left(\frac{j+1}{j+2}\right)^{2}\left(L_{j+1}(x)+L_{j+2}(x)\right) ; \\
& \psi_{j}(x):=L_{j}(x)-\frac{j}{j+2} L_{j+1}(x) .
\end{aligned}
$$

Since $L_{l}(-1)=(-1)^{l}$ and $L_{l}^{\prime}(1)=\frac{1}{2} l(l+1)$, one can verify easily that

$$
\phi_{j}(-1)=\phi_{j}^{\prime}(1)=\psi_{j}^{\prime}(1)=0 .
$$

Hence, for $n=1$ or $n=2$ with $m>0, W_{N}^{(m, n)}=\operatorname{span}\left\{\phi_{j}: j=0,1, \ldots, N-2\right\}$; and for $n=3$ or $n=2$ with $m=0, W_{N}^{(m, n)}=\operatorname{span}\left\{\psi_{j}: j=0,1, \ldots, N-1\right\}$.

Now, let us write

$$
v_{N}(r):=w_{N}^{R}(x)+\mathrm{i} w_{N}^{I}(x), \quad 2^{n-3} r^{n-1} h(r):=q^{R}(x)+\mathrm{i} q^{I}(x),
$$

where $w_{N}^{R}, w_{N}^{I}, q^{R}$, and $q^{I}$ are real functions in $\hat{I}$. Our spectral-Galerkin algorithm is to seek $w_{N}^{R}, w_{N}^{I} \in W_{N}$ such that for any real polynomials $\phi, \psi \in W_{N}$,

$$
\begin{gathered}
\left((1+x)^{n-1} \partial_{x} w_{N}^{R}, \partial_{x} \phi\right)+d_{m}\left((1+x)^{n-3} w_{N}^{R}, \phi\right)+k\left((1+x)^{n-1} \partial_{x} w_{N}^{I}, \phi\right) \\
+\frac{n-1}{2} k\left((1+x)^{n-2} w_{N}^{I}, \phi\right)=\left(q^{R}, \phi\right) ; \\
\left((1+x)^{n-1} \partial_{x} w_{N}^{I}, \partial_{x} \psi\right)+d_{m}\left((1+x)^{n-3} w_{N}^{I}, \psi\right)-k\left((1+x)^{n-1} \partial_{x} w_{N}^{R}, \psi\right) \\
-\frac{n-1}{2} k\left((1+x)^{n-2} w_{N}^{R}, \phi\right)=\left(q^{I}, \psi\right) .
\end{gathered}
$$

Thanks to the nice properties of the Legendre polynomials, one can find that the coefficient matrix of the above system is sparse, and its nonzero entries can be determined exactly.

4.2.2. Numerical results. We present some numerical results for the problem (2.1)-(2.3) by using the schemes proposed above.

Example 1. We consider (2.1)-(2.3) with $n=2, d_{m}=100$, and $g=0$ and set the exact solution to be

$$
u(r)=v(r) e^{\mathrm{i} k r}, \quad r \in I,
$$

where $v(r)=(\cos 2 k-\cos (2 k(1-r)))+\mathrm{i}\left(\frac{1}{k}(\sin 2 k-\sin (2 k(1-r)))-2 r \cos (2 k(1-r))\right)$ is the exact solution of the transformed problem (4.2). 

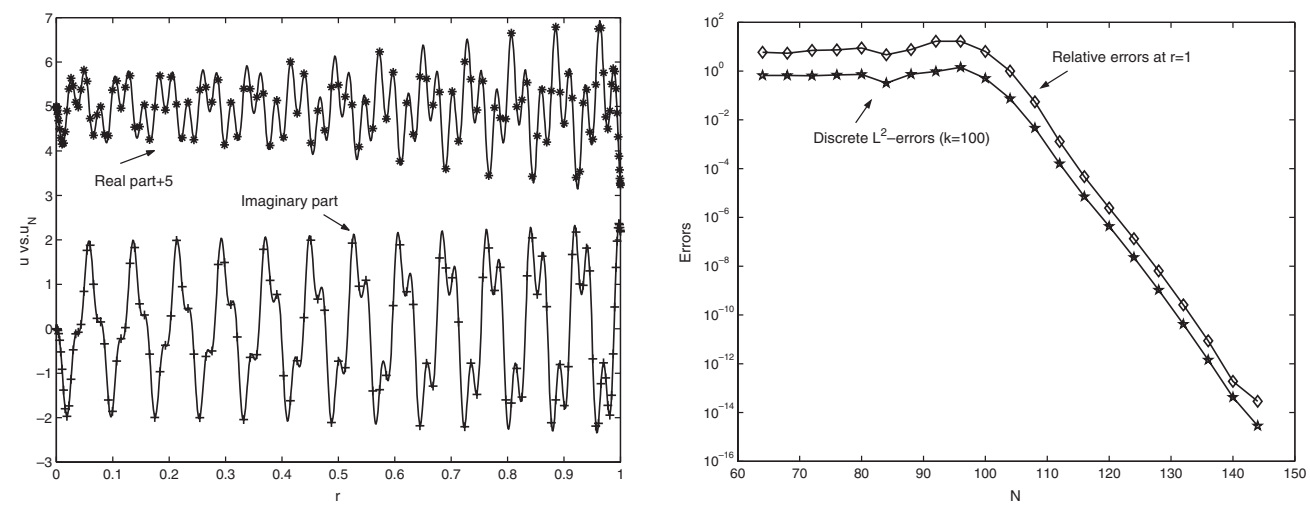

FiG. 4.1. Left: exact solution vs. numerical solution. Right: errors vs. $N(k=100)$.

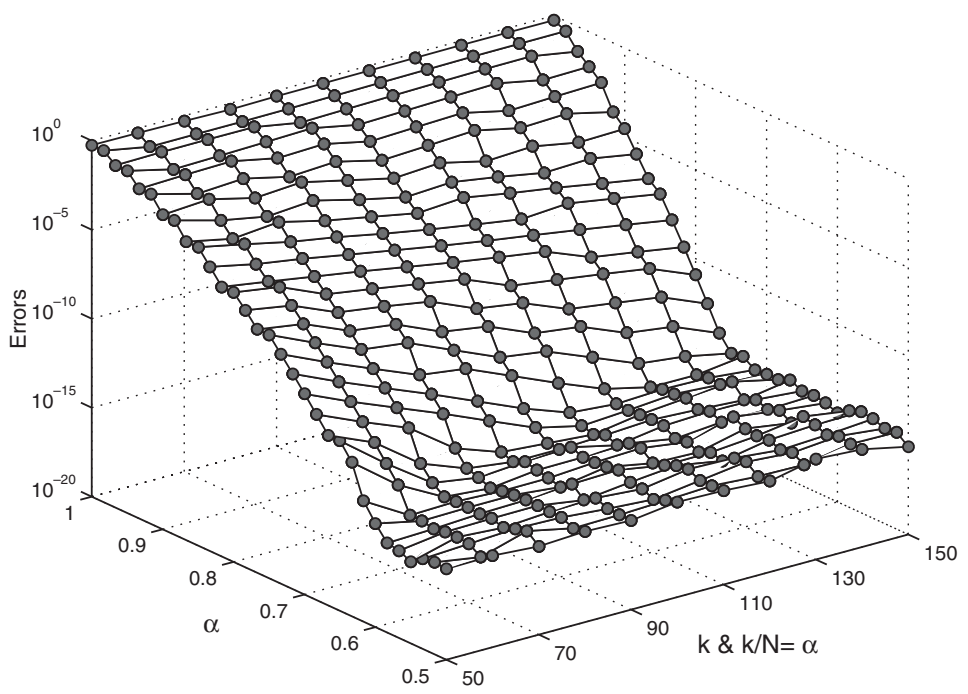

FIG. 4.2. Errors vs. $\alpha \in[0.5,1]$ and $k \in[50,150]$ with $\frac{k}{N}=\alpha$.

In Figure 4.1 (left), we plot the numerical solution at Legendre-Gauss-Lobatto points with $k=80$ and $N=96$ (asterisk-markers for the real part (raised by 5 unit) and plus-markers for the imaginary part) vs. the exact solution (solid line).

We now examine the convergence rate. According to Theorem 3.3, the predicted order of convergence for the exact solution (4.21) is

$$
\left\|u-u_{N}\right\|_{\omega} \sim k^{1+s} N^{1-s}, \quad N \gg 1, \quad k>0, s \geq 1 .
$$

In Figure 4.1 (right), we fix the wave number $k=100$ and plot the discrete $L^{2}$ errors and relative errors at $r=1$ vs. different modes $N$. As expected, an exponential convergence rate is observed once $N$ is large enough to resolve the oscillation.

Next, we fix $\alpha=\frac{k}{N}$ and examine the error behavior with respect to $\alpha$. In Figure 4.2 , we plot the discrete $L^{2}$-errors with $0.5 \leq \alpha \leq 1,50 \leq k \leq 150$, and $N=\frac{k}{\alpha}$. The results indicate that the proposed scheme can provide very accurate approximations to highly oscillatory solutions under the condition $\frac{k}{N}=\alpha<1$, which is necessary for 

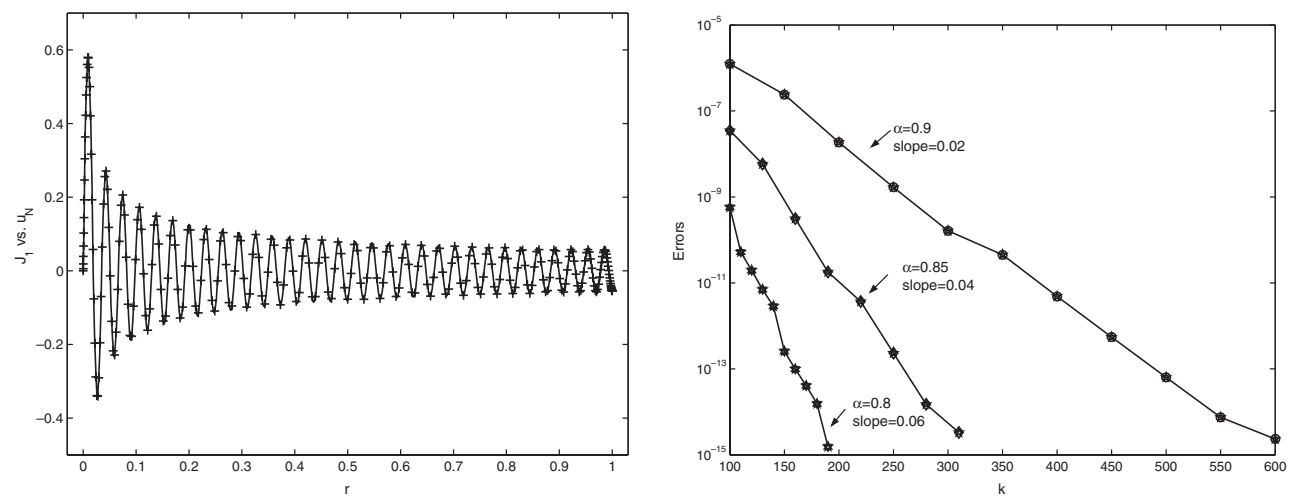

FIG. 4.3. Left: exact solution vs. numerical solution. Right: errors vs. wave number $k$ with $\alpha=\frac{k}{N}$ fixed.

convergence (cf. [13]).

Example 2. We consider the problem (2.1)-(2.3) with $n=2$ and $d_{m}=1$. An exact solution is

$$
u(r)=J_{1}(k r), \quad \text { with } f \equiv 0 \text { and } g=k\left(J_{1}^{\prime}(k)-\mathrm{i} J_{1}(k)\right),
$$

where $J_{1}(\cdot)$ is the first degree Bessel function of the first kind. As pointed out in [26], we have the following asymptotic property:

$$
u(r)=J_{1}(k r)=\sqrt{\frac{2}{\pi k r}} \cos \left(k r-\frac{3}{4} \pi\right)+O\left((k r)^{-\frac{3}{2}}\right) \quad \text { if } k r \gg 1 .
$$

Hence, the solution is highly oscillating when the wave number $k$ is large (see Figure 4.3 (left)). We derive from (4.24) that the expected convergence rate is $k^{\frac{1}{2}+s} N^{1-s}$. In Figure 4.3 (left), we plot the exact solution vs. the numerical solution with $k=200$ and $N=256$. In this case, the discrete $L^{2}$-error is $2.45 \times 10^{-15}$ and relative error at $r=1$ is $3.84 \times 10^{-13}$. The error behaviors with several fixed $\alpha=\frac{k}{N}$ are plotted in Figure 4.3 (right), which demonstrates that the spectral-Galerkin method is capable of providing very accurate results even for $\alpha$ close to 1 .

5. Extensions to multidimensional cases. The results we derived for the prototypical 1-D problem (2.1)-(2.3) (with $n=2,3$ ) can be used to derive error estimates for the spectral-Galerkin approximation to the multidimensional problem (1.2). As an example, we consider the case $n=3$ :

$$
\begin{aligned}
-\Delta U-k^{2} U=F & \text { in } \hat{\Omega}:=\left\{(x, y, z): a^{2}<x^{2}+y^{2}+z^{2}<b^{2}\right\}, \\
\partial_{r} U-\mathrm{i} k U=G & \text { on } S_{b}:=\left\{(x, y, z): x^{2}+y^{2}+z^{2}=b^{2}\right\}, \\
U=0 & \text { on } \quad S_{a}:=\left\{(x, y, z): x^{2}+y^{2}+z^{2}=a^{2}\right\} \text { if } a>0 .
\end{aligned}
$$

Applying the spherical transformation

$$
x=r \cos \theta \sin \phi, \quad y=r \sin \theta \sin \phi, \quad z=r \cos \phi
$$


to (5.1) and setting $u(r, \theta, \phi)=U(x, y, z), f(r, \theta, \phi)=F(x, y, z), g(\theta, \phi)=G(x, y, z)$, and $S:=[0,2 \pi) \times[0, \pi)$, we obtain

$$
\begin{aligned}
-\left(\frac{\partial^{2}}{\partial r^{2}}+\frac{2}{r} \frac{\partial}{\partial r}+\frac{1}{r^{2}} \Delta_{S}\right) u-k^{2} u & =f & & \text { in } \Omega:=(a, b) \times S, \\
\partial_{r} u-\mathrm{i} k u & =g & & \text { on } S_{b}, \\
u & =0 & & \text { on } S_{a} \text { if } a>0,
\end{aligned}
$$

where $\Delta_{S}$ is the Laplace-Beltrami operator (the Laplacian on the unit sphere $S$ ):

$$
\Delta_{S}=\frac{1}{\sin ^{2} \phi} \frac{\partial^{2}}{\partial \theta^{2}}+\frac{\cos \phi}{\sin \phi} \frac{\partial}{\partial \phi}+\frac{\partial^{2}}{\partial^{2} \phi}
$$

We recall that the spherical harmonic functions $\left\{Y_{l, m}\right\}$ are the eigenfunctions of the Laplace-Beltrami operator (see [25])

$$
-\Delta_{S} Y_{l, m}(\theta, \phi)=m(m+1) Y_{l, m}(\theta, \phi)
$$

and are defined by

$$
Y_{l, m}(\theta, \phi)=\sqrt{\frac{(2 m+1)(m-l) !}{4 \pi(m+l) !}} e^{i l \theta} P_{m}^{l}(\cos \phi), \quad m \geq|l| \geq 0,
$$

where $P_{m}^{l}(x)$ is the associated Legendre functions given by

$$
P_{m}^{l}(x)=\frac{(-1)^{l}}{2^{m} m !}\left(1-x^{2}\right)^{\frac{l}{2}} \frac{d^{m+l}}{d x^{m+1}}\left\{\left(x^{2}-1\right)^{m}\right\} .
$$

The set of harmonic functions forms a complete orthonormal system in $L^{2}(S)$, i.e.,

$$
\int_{0}^{2 \pi} \int_{0}^{\pi} Y_{l, m}(\theta, \phi) \overline{Y_{l^{\prime}, m^{\prime}}}(\theta, \phi) \sin \phi d \phi d \theta=\delta_{l, l^{\prime}} \delta_{m, m^{\prime}}
$$

Hence, for any function $U(x, y, z) \in L^{2}(\hat{\Omega})$, the function $u(r, \theta, \phi)=U(x, y, z)$ can be expanded as

$$
u=\sum_{|l|=0}^{\infty} \sum_{m \geq|l|}^{\infty} u_{l m}(r) Y_{l, m}(\theta, \phi), \text { with } u_{l m}(r)=\int_{S} u(r, \theta, \phi) \bar{Y}_{l, m}(\theta, \phi) d S,
$$

and we have

$$
\|u\|_{L_{\omega^{2}}^{2}(\Omega)}^{2}=\sum_{|l|=0}^{\infty} \sum_{m \geq|l|}^{\infty}\left\|u_{l m}\right\|_{\omega^{2}}^{2}=\|U\|_{L^{2}(\hat{\Omega})}^{2} \quad\left(\omega^{2}=r^{2}\right) .
$$

For a scalar function $v$ on $S$, the gradient operator $\vec{\nabla}_{S}$ on the unit sphere is defined by $\vec{\nabla}_{S} v=\left(\frac{1}{\sin \phi} \partial_{\theta} v, \partial_{\phi} v\right)$. One can verify readily that

$$
-\left(\Delta_{S} u, v\right)_{S}=\left(\vec{\nabla}_{S} u, \vec{\nabla}_{S} v\right)_{S} \quad \forall u, v \in \mathcal{D}\left(\Delta_{S}\right),
$$

where $\mathcal{D}\left(\Delta_{S}\right)$ is the domain of the Laplace-Beltrami operator $\Delta_{S}$. In particular, as a consequence of (5.5)-(5.9), we have

$$
\left(\vec{\nabla}_{S} Y_{l, m}, \vec{\nabla}_{S} Y_{l, m}\right)_{S}=m(m+1), \quad m \geq|l| \geq 0 .
$$


Accordingly, we can define the Sobolev space on $S$ :

$$
H^{1}(S):=\left\{u: u \text { is measurable on } S \text { and }\|u\|_{H^{1}(S)}^{2}<\infty\right\},
$$

where $\|u\|_{H^{1}(S)}=\left(\|u\|_{L^{2}(S)}^{2}+\left\|\vec{\nabla}_{S} u\right\|_{L^{2}(S)}^{2}\right)^{\frac{1}{2}}$.

The variational formulation of (5.3) is to find $u \in V:=H_{\omega^{2}}^{1}\left(I ; L^{2}(S)\right) \cap L^{2}\left(I ; H^{1}(S)\right)$ such that $\left(\omega^{2}=r^{2}\right)$

$$
\begin{aligned}
a(u, v) & :=\left(\partial_{r} u, \partial_{r} v\right)_{\omega^{2}, \Omega}+\left(\vec{\nabla}_{S} u, \vec{\nabla}_{S} v\right)_{\Omega}-k^{2}(u, v)_{\omega^{2}, \Omega} \\
& -\mathrm{i} k b^{2}(u(b, \cdot), v(b, \cdot))_{S}=(f, v)_{\omega^{2}, \Omega}+b^{2}(g, v(b, \cdot))_{S} \quad \forall v \in V .
\end{aligned}
$$

The spectral-Galerkin approximation of (5.11) is to find $u_{M N} \in V_{M N}$ such that

$$
a\left(u_{M N}, v\right)=(f, v)_{\omega^{2}, \Omega}+b^{2}(g, v(b, \cdot))_{S} \quad \forall v \in V_{M N},
$$

where $V_{M N}:=W_{M} \times X_{N}$, and

$$
W_{M}:=\operatorname{span}\left\{Y_{l, m}: 0 \leq|l| \leq m \leq M\right\}, X_{N}:=\left\{u \in P_{N}: u(a)=0 \text { if } a>0\right\} .
$$

Hence, we can write

$$
\begin{gathered}
(u(r, \theta, \phi), f(r, \theta, \phi), g(\theta, \phi))=\sum_{|l|=0}^{\infty} \sum_{m \geq|l|}^{\infty}\left(u_{l m}(r), f_{l m}(r), g_{l m}\right) Y_{l, m}(\theta, \phi) ; \\
u_{M N}(r, \theta, \phi)=\sum_{|l|=0}^{M} \sum_{m \geq|l|}^{M} u_{l m}^{N}(r) Y_{l, m}(\theta, \phi) .
\end{gathered}
$$

In order to describe the error bounds, we define a nonisotropic space $\widetilde{H}_{\omega^{2}}^{s}\left(I ; H^{t}(S)\right)$ as follows:

$$
\widetilde{H}_{\omega^{2}}^{s}\left(I ; H^{t}(S)\right)=\left\{u \in L_{\omega^{2}}^{2}(\Omega): \sum_{|l|=0}^{\infty} \sum_{m \geq|l|}^{\infty} m^{t}(m+1)^{t}\left\|u_{l m}\right\|_{\widetilde{H}_{\omega^{2}}^{s}(I)}^{2}<+\infty\right\}
$$

where $\left\{u_{l m}\right\}$ are the expansion coefficients of $u$ in terms of $Y_{l, m}$ as in (5.7). Thanks to $(5.10)$, we can define the norm on $\widetilde{H}_{\omega^{2}}^{s}\left(I ; H^{t}(S)\right)$ by

$$
\|u\|_{\widetilde{H}_{\omega^{2}}^{s}\left(I ; H^{t}(S)\right)}=\left(\sum_{|l|=0}^{\infty} \sum_{m \geq|l|}^{\infty} m^{t}(m+1)^{t}\left\|u_{l m}\right\|_{\widetilde{H}_{\omega^{2}}^{s}(I)}^{2}\right)^{\frac{1}{2}}
$$

and its seminorm by replacing $\left\|u_{l m}\right\|_{\widetilde{H}_{\omega^{2}}^{s}(I)}$ with $\left|u_{l m}\right|_{\widetilde{H}_{\omega^{2}}^{s}(I)}$. In particular, $L_{\omega^{2}}^{2}\left(I ; H^{t}(S)\right)$ $=\widetilde{H}_{\omega^{2}}^{0}\left(I ; H^{t}(S)\right)$ and $\widetilde{H}_{\omega^{2}}^{s}\left(I ; L^{2}(S)\right) \stackrel{\omega^{2}}{=} \widetilde{H}_{\omega^{2}}^{s}\left(I ; H^{0}(S)\right)$.

5.1. In a sphere $(\boldsymbol{a}=\mathbf{0})$. Without loss of generality, we assume that $b=1$. In this case, we can show that $\left\{u_{l m}\right\}$ (resp., $\left\{u_{l m}^{N}\right\}$ ) satisfy the 1-D problem (2.5) (resp., (3.1)) with $n=3$ and $f, g$ being replaced by $f_{l m}$ and $g_{l m}$, respectively.

THEOREM 5.1. Let $u$ and $u_{M N}$ be, respectively, the solutions of (5.11) and (5.12), and denote $e=u-u_{M N}$. Then if

$$
u \in L^{2}\left(I ; H^{t}(S)\right) \cap H_{\omega^{2}}^{1}\left(I ; H^{t-1}(S)\right) \cap \widetilde{H}_{\omega^{2}}^{s}\left(I ; L^{2}(S)\right), \quad s, t \geq 1, s, t \in \mathbb{N}
$$


we have

$$
\begin{aligned}
\left\|\partial_{r} e\right\|_{L_{\omega^{2}}^{2}(\Omega)} & +\left\|\vec{\nabla}_{S} e\right\|_{L^{2}(\Omega)}+k\|e\|_{L_{\omega^{2}}^{2}(\Omega)} \\
& \lesssim C_{*}\left(\left(M+M^{4} k^{-4}+k^{2} N^{-1}\right) N^{1-s}+M^{1-t}\left(1+k M^{-1}\right)\right),
\end{aligned}
$$

where $C_{*}$ is a positive constant depending only on the seminorms of $u$ in the spaces mentioned in (5.16).

Proof. Let $e_{l m}(r)=u_{l m}(r)-u_{l m}^{N}(r)$. We deduce from Theorem 3.2 that

$$
\begin{aligned}
& \left\|\partial_{r} e_{l m}\right\|_{L_{\omega^{2}}^{2}(I)}+\sqrt{d_{m}}\left\|e_{l m}\right\|_{L^{2}(I)}+k\left\|e_{l m}\right\|_{L_{\omega^{2}}^{2}(I)} \\
& \lesssim\left(1+\sqrt{d_{m}}+d_{m}^{2} k^{-4}+k^{2} N^{-1}\right) N^{1-s}\left|e_{l m}\right|_{\widetilde{H}_{\omega^{2}}^{s}(I)},
\end{aligned}
$$

where $d_{m}=m(m+1)$. Therefore, by (5.6)-(5.10) and (5.13b)-(5.14),

$$
\begin{aligned}
\left\|\partial_{r} e\right\|_{L_{\omega^{2}}^{2}(\Omega)}^{2}+\left\|\vec{\nabla}_{S} e\right\|_{L^{2}(\Omega)}^{2}+k^{2}\|e\|_{L_{\omega^{2}}^{2}(\Omega)}^{2} & \sum_{|l|=0}^{M} \sum_{m \geq|l|}^{M}\left(\left\|\partial_{r} e_{l m}\right\|_{L_{\omega^{2}}^{2}(I)}^{2}+d_{m}\left\|e_{l m}\right\|_{L^{2}(I)}^{2}+k^{2}\left\|e_{l m}\right\|_{L_{\omega^{2}}^{2}(I)}^{2}\right) \\
& +\left(\sum_{|l|=0}^{\infty} \sum_{m>M}^{\infty}+\sum_{|l|>M}^{\infty} \sum_{m \geq|l|}^{\infty}\right)\left(\left\|\partial_{r} u_{l m}\right\|_{L_{\omega^{2}}^{2}(I)}^{2}+d_{m}\left\|u_{l m}\right\|_{L^{2}(I)}^{2}+k^{2}\left\|u_{l m}\right\|_{L_{\omega^{2}}^{2}(I)}^{2}\right) \\
\lesssim & \left(1+\sqrt{d_{M}}+d_{M}^{2} k^{-4}+k^{2} N^{-1}\right)^{2} N^{2-2 s} \sum_{|l|=0}^{M} \sum_{m \geq|l|}^{M}\left|u_{l m}\right|_{\widetilde{H}_{\omega^{2}}^{s}(I)}^{2} \\
& +d_{M}^{1-t} \sum_{|l|=0}^{\infty} \sum_{m \geq|l|}^{\infty}\left(d_{m}^{t-1}\left(\left\|\partial_{r} u_{l m}\right\|_{L_{\omega^{2}}^{2}(I)}^{2}+d_{m}\left\|u_{l m}\right\|_{L^{2}(I)}^{2}+k^{2}\left\|u_{l m}\right\|_{L_{\omega^{2}}^{2}(I)}^{2}\right)\right) \\
\lesssim & \left(M+M k^{-4}+k^{2} N^{-1}\right)^{2} N^{2-2 s}|u|_{\widetilde{H}_{\omega^{2}}^{s}\left(I ; L^{2}(S)\right)}^{2} \\
& +d_{M}^{1-t}\left(|u|_{H_{\omega^{2}}^{1}\left(I ; H^{t-1}(S)\right)}^{2}+|u|_{L^{2}\left(I ; H^{t}(S)\right)}^{2}+k^{2} d_{m}^{-1}|u|_{L_{\omega^{2}}^{2}\left(I ; H^{t}(S)\right)}^{2}\right)
\end{aligned}
$$

which implies the desired result.

5.2. In a spherical shell $(\boldsymbol{a}>\mathbf{0})$. In this case, $\left\{u_{l m}\right\}$ are the solutions of

$$
\widehat{\mathcal{B}}_{l m}\left(u_{l m}, v\right)=\left(f_{l m}, v\right)_{\omega^{2}}+b^{2} g_{l m} \overline{v(b)} \quad \forall v \in X, \quad 0 \leq|l| \leq m
$$

where $X:=\left\{u \in H^{1}(I): u(a)=0\right\}$, and

$$
\widehat{\mathcal{B}}_{l m}(u, v):=\left(\partial_{r} u, \partial_{r} v\right)_{\omega^{2}}+d_{m}(u, v)-k^{2}(u, v)_{\omega^{2}}-\mathrm{i} k b^{2} u(b) \overline{v(b)},
$$

with $\omega^{2}=r^{2}, d_{m}=m(m+1)$. The numerical approximations $u_{l m}^{N}(0 \leq|l| \leq m, m=$ $0,1, \ldots, M)$ are defined by

$$
\widehat{\mathcal{B}}_{l m}\left(u_{l m}^{N}, v_{N}\right)=\left(f_{l m}, v_{N}\right)_{\omega^{2}}+b^{2} g_{l m} \overline{v_{N}(b)} \quad \forall v_{N} \in X_{N}:=X \cap P_{N} .
$$

Since $u_{l m},(r-a) u_{l m} \in X$ (resp., $\left.u_{l m}^{N},(r-a) u_{l m}^{N} \in X_{N}\right)$, we can use them as test functions in (5.19) (resp., (5.21)), and derive the following results using an argument analogous to that in the proof of Theorem 2.2 . 
LEMma 5.1. Let $\left\{u_{l m}\right\}$ and $\left\{u_{l m}^{N}\right\}$ be, respectively, the solution of (5.19) and (5.21). Then there exists $\xi \in(a, b)$ such that for $C_{\xi}:=\left(2-\frac{2 a}{\xi}\right)^{-1}$, we have

$$
\begin{aligned}
& \left\|\partial_{r} u_{l m}\right\|_{L_{\omega^{2}}^{2}(I)}^{2}+d_{m}\left\|u_{l m}\right\|_{L^{2}(I)}^{2}+k^{2}\left\|u_{l m}\right\|_{L_{\omega^{2}}^{2}(I)}^{2} \lesssim C_{\xi} b^{3}\left(\left|g_{l m}\right|^{2}+b^{2}\left\|f_{l m}\right\|_{L_{\omega^{2}}^{2}(I)}^{2}\right), \\
& \left\|\partial_{r} u_{l m}^{N}\right\|_{L_{\omega^{2}}^{2}(I)}^{2}+d_{m}\left\|u_{l m}^{N}\right\|_{L^{2}(I)}^{2}+k^{2}\left\|u_{l m}^{N}\right\|_{L_{\omega^{2}}^{2}(I)}^{2} \lesssim C_{\xi} b^{3}\left(\left|g_{l m}\right|^{2}+b^{2}\left\|f_{l m}\right\|_{L_{\omega^{2}}^{2}(I)}^{2}\right) .
\end{aligned}
$$

The above a priori estimates allow us to perform the error analysis for the spherical shell case. Similar to the case $a=0$, we can prove the following.

THEOREM 5.2. Let $u$ and $u_{M N}$ be, respectively, the solutions of (5.11) and (5.12), and denote $e=u-u_{M N}$. Then if

$u \in L^{2}\left((a, b) ; H^{t}(S)\right) \cap H_{\omega^{2}}^{1}\left((a, b) ; H^{t-1}(S)\right) \cap \widetilde{H}_{\omega^{2}}^{s}\left((a, b) ; L^{2}(S)\right), \quad s, t \geq 1, s, t \in \mathbb{N}$, there exists $\xi \in(a, b)$ such that for $C_{\xi}:=\left(2-\frac{2 a}{\xi}\right)^{-1}$, we have

$$
\begin{aligned}
\left\|\partial_{r} e\right\|_{L_{\omega^{2}}^{2}(\Omega)} & +\left\|\vec{\nabla}_{S} e\right\|_{L^{2}(\Omega)}+k\|e\|_{L_{\omega^{2}}^{2}(\Omega)} \\
& \lesssim C_{*} b^{2}\left(1+\sqrt{C_{\xi}}\right)\left(\left(M+M^{4} k^{-4}+k^{2} N^{-1}\right) N^{1-s}+M^{1-t}\left(1+k M^{-1}\right)\right),
\end{aligned}
$$

where $C_{*}$ is a positive constant depending only on the seminorms of $u$ in the spaces mentioned in (5.16).

Remark 5.1. A similar procedure can be performed for the Helmholtz equation (1.2) in a 2-D axisymmetric domain $(n=2)$ by using a Fourier expansion in the $\theta$-direction.

6. Concluding remarks. We presented in this paper a complete error analysis and an efficient numerical algorithm for the spectral-Galerkin approximation of the Helmholtz equation with high wave numbers in a 1-D domain as well as in multidimensional radial and spherical symmetric domains.

Our analysis is made possible by using two new arguments: (i) we employed a new procedure advocated in [7] which allowed us to obtain sharp (in terms of $k$ ) a priori estimates for both the continuous and discrete problems; (ii) we used new Jacobi and generalized Jacobi approximation results developed recently in $[16,24]$ which enabled us to derive optimal estimates for the cases $n=2,3$ which involve degenerate/singular coefficients.

Unlike in most of the previous studies on the approximation of the Helmholtz equation with high wave numbers, our analysis does not rely on explicit knowledge of continuous/discrete Green's functions and is valid without any restriction on the wave number $k$ and the discretization parameter $N$. Hence, it is possible to extend our results to more complex problems such as Helmholtz equations in an inhomogeneous medium and to more complex domains through a suitable mapping or a domain perturbation technique.

Acknowledgment. The authors would like to thank Dr. Xiaobing Feng for many stimulating discussions and helpful suggestions.

\section{REFERENCES}

[1] I. M. BABUŠKa AND S. A. SAUter, Is the pollution effect of the FEM avoidable for the Helmholtz equation considering high wave numbers?, SIAM J. Numer. Anal., 34 (1997), pp. 23922423. 
[2] J. P Berenger, A perfectly matched layer for the absorption of electromagnetic waves, J. Comput. Phys., 114 (1994), pp. 185-200.

[3] C. Bernardi and Y. Maday, Spectral methods, in Handbook of Numerical Analysis, Vol. 5 (Part 2), P. G. Ciarlet and L. L. Lions, eds., North-Holland, Amsterdam, 1997, pp. 209-485.

[4] C. Canuto, M. Y. Hussaini, A. Quarteroni, and T. A. Zang, Spectral Methods in Fluid Dynamics, Springer-Verlag, New York, 1988.

[5] R. D. Ciskowski and C. A. Brebbia, Boundary Element Methods in Acoustics, Elsevier, London, 1991.

[6] P. Cummings, Analysis of Finite Element Based Numerical Methods for Acoustic Waves, Elastic Waves, and Fluid-Solid Iterations in the Frequency Domain, Ph.D. thesis, University of Tennessee, Knoxville, 2001.

[7] P. Cummings And X. B. Feng, Sharp regularity coefficient estimates for complex-valued acoustic and elastic Helmholtz equations, Math. Models Methods Appl. Sci., to appear.

[8] J. Douglas, J. E. Santos, D. Sheen, and L. S. Bennethum, Frequency domain treatment of one-dimensional scalar waves, Math. Models Methods Appl. Sci., 3 (1993), pp. 171-194.

[9] J. Douglas, JR., D. Sheen, And J. E. Santos, Approximation of scalar waves in the spacefrequency domain, Math. Models Methods Appl. Sci., 4 (1994), pp. 509-531.

[10] X. Feng And D. Sheen, An elliptic regularity coefficient estimate for a problem arising from a frequency domain treatment of waves, Trans. Amer. Math. Soc., 346 (1994), pp. 475-487.

[11] K. Gerdes And L. Demkowicz, Solution of 3D-Laplace and Helmholtz equations in exterior domains using hp-infinite elements, Comput. Methods Appl. Mech. Engrg., 137 (1996), pp. 239-273.

[12] K. Gerdes and F. Ihlenburg, On the pollution effect in FE solutions of the 3D-Helmholtz equation, Comput. Methods Appl. Mech. Engrg., 170 (1999), pp. 155-172.

[13] D. Gottlieb and S. A. Orszag, Numerical Analysis of Spectral Methods: Theory and Applications, CBMS-NSF Regional Conf. Ser. in Appl. Math. 26, SIAM, Philadelphia, 1977.

[14] M. J. Grote and J. B. Keller, On non-reflecting boundary conditions, J. Comput. Phys., 122 (1995), pp. 231-243.

[15] B. Y. GUO AND L. L. WANG, Jacobi interpolation approximations and their applications to singular differential equations, Adv. Comput. Math., 14 (2001), pp. 227-276.

[16] B. Y. Guo AND L. L. WANG, Jacobi approximations in non-uniformly Jacobi-weighted Sobolev spaces, J. Approx. Theory, 128 (2004), pp. 1-41.

[17] G. H. Hardy, J. E. Littlewood, and G. Pólya, Inequalities, Cambridge University Press, Cambridge, UK, 1952.

[18] F. Ihlenburg and I. BABUŠKa, Finite element solution of the Helmholtz equation with high wave number, Part I: The h-version of FEM, Comput. Math. Appl., 30 (1995), pp. 9-37.

[19] F. Ihlenburg and I. BABUŠKa, Finite element solution of the Helmholtz equation with high wave number. Part II: The h-p version of the FEM, SIAM J. Numer. Anal., 34 (1997), pp. $315-358$.

[20] F. John, Partial Differential Equations, 4th ed., Springer-Verlag, New York, 1982.

[21] A. H. Schatz, An observation concerning Ritz-Galerkin methods with indefinite bilinear forms, Math. Comp., 28 (1974), pp. 959-962.

[22] J. SHEn, Efficient spectral-Galerkin method. I. Direct solvers for second-and fourth-order equations using Legendre polynomials, SIAM J. Sci. Comput., 15 (1994), pp. 1489-1505.

[23] J. Shen, Efficient spectral-Galerkin methods III: Polar and cylindrical geometries, SIAM J. Sci. Comput., 18 (1997), pp. 1583-1604.

[24] J. Shen And L. L. WANG, Error analysis for mapped Jacobi spectral methods, J. Sci. Comput., to appear.

[25] I. N. Sneddon, Special Functions of Mathematical Physics and Chemistry, 3rd ed., Longman, New York, 1980.

[26] G. N. Watson, A Treatise of the Theory of Bessel Functions, 2nd ed., Cambridge University Press, Cambridge, UK, 1966. 